\title{
ESPECIAÇÃO DINÂMICA DE METAIS TRAÇO EM AMBIENTE AQUÁTICO USANDO CRONOPOTENCIOMETRIA DE REDISSOLUÇÃO ANÓDICA
}

\author{
Adnívia Santos Costa Monteiro ${ }^{a, c, \#}$, Danielle Goveia ${ }^{b}$, Elise Rotureau ${ }^{\mathrm{d}}$, André Henrique Rosa ${ }^{\mathrm{a}, *}$, Jorge Cesar Masini ${ }^{\mathrm{c}}$ e José \\ Paulo Pinheiro ${ }^{\mathrm{d}}$ \\ anstituto de Ciência e Tecnologia, Universidade Estadual Paulista, 18087-180 Sorocaba - SP, Brasil \\ ${ }^{b}$ Universidade Estadual Paulista, Campus de Itapeva, 18409-010 Itapeva - SP, Brasil \\ 'Departamento de Química Fundamental, Instituto de Química, Universidade de São Paulo, Av. Prof. Lineu Prestes 748, 05508-000 \\ São Paulo- SP, Brasil \\ dLIEC/UMR7360, Université de Lorraine, CNRS, 15 Avenue du Charmois, Vandoeuvre-lès-Nancy F-54501, França
}

Recebido em 26/01/2018; aceito em 12/04/2018; publicado na web em 22/05/2018

\begin{abstract}
DYNAMIC SPECIATION OF TRACE METALS IN AQUATIC ENVIRONMENT USING ANODIC STRIPPING CHRONOPOTENTIOMETRY. The contamination of natural environments by trace metals has several adverse effects on human health, which is mainly due to the high toxicity, persistence and reactivity of these species in the environment. To understand the bioavailability of trace metals, it is necessary to know the speciation in equilibrium and the dynamic behavior of the system. In this context, the present work aims to present a bibliographic review about the potentialities of the Stripping Chronopotentiometry (SCP) and Stripping Chronopotentiometry at Scanned Deposition Potential (SSCP) in the study of dynamic trace metal speciation. These techniques have low detection limits and do not suffer interference due to the adsorption of organic matter on the surface of the working electrode. Coupling these techniques with the Absence of Gradients and Nernstian Equilibrium Stripping (AGNES) enables simultaneous determination of free ions, complexation equilibrium constants and diffusion coefficients of the complexes, thus providing information on the dynamic and heterogeneity of the metal complexes in the presence of macromolecules or particles. In this paper we will present the theory of dynamic speciation of trace metals, a detailed description of how to use the techniques to obtain this information, and examples of application in model systems and natural samples.
\end{abstract}

Keywords: metal complexes; metal ions; lability; redissolution techniques; deposition time.

\section{INTRODUÇÃO}

O interesse no estudo de metais traço em solução provém da elevada toxicidade, persistência e reatividade de muitos destes no ambiente. Em ambientes aquáticos naturais, os íons metálicos podem se distribuir em diferentes espécies como resultado das interações com componentes da matriz (matéria orgânica, partículas inorgânicas) e das características físico-químicas (temperatura, força iônica, $\mathrm{pH}$, potencial redox, presença de outros íons) do meio. A maioria das espécies formadas tem caráter polifuncional e polieletrolítico, apresentando ampla faixa de constantes de velocidade de formação/ dissociação e de tamanhos, que controlam a biodisponibilidade, toxicidade e mobilidade dos íons metálicos. ${ }^{1}$

O estudo da distribuição dessas espécies é denominado de especiação de metais. O termo especiação foi definido como a descrição da abundância de um elemento numa amostra (distribuição da espécie), enquanto a análise de especiação se refere aos procedimentos analíticos de identificação e/ou medida quantitativa de uma ou mais espécies químicas numa amostra. ${ }^{2}$

Cada uma destas espécies pode se comportar de forma diferente em termos de mobilidade e biodisponibilidade em sistemas aquáticos naturais, portanto, estão sujeitas à mudança de condições e praticamente nunca atingem o equilíbrio químico. Isto torna o estudo da especiação muito mais complicado, uma vez que se faz necessária uma compreensão da cinética das reações do íon metálico para elucidar as relações entre as suas diferentes formas físico-químicas, suas reatividades, mobilidade e biodisponibilidades nos processos ambientais e biológicos. ${ }^{3}$

*e-mail: ahrosa@unesp.com.br

\#e-mail alternativo: adniviacosta@hotmail.com
Em águas naturais, a concentração total de metais relevantes para o ambiente é geralmente baixa $(<100 \mathrm{nM})$, sendo que para íons metálicos livres é frequentemente muito mais baixa $(<1 \mathrm{nM})$. Desta forma, técnicas analíticas devem combinar capacidade de especiação e alta sensibilidade. ${ }^{4}$

Do ponto de vista experimental o número de técnicas capazes de determinar a concentração de íons metálicos livres e de realizar estudos de especiação dinâmica aos níveis de concentrações de metais traço em águas naturais é muito limitado. As duas revisões mais relevantes sobre esse assunto, publicadas em 2006 por Sigg et $a l .,{ }^{5}$ e 2009 por Pesavento et al., ${ }^{6}$ classificam as técnicas analíticas para especiação em águas naturais em técnicas eletroanalíticas e de separação. As técnicas eletroanalíticas incluem a voltametria de redissoluçao anodica (ASV), a cronopotenciometria de redissolução anódica (SCP), a voltametria de redissolução catódica com competição de ligante (CLE-SV) e a técnica de redissolução em equilíbrio nernstiano na ausência de gradientes (AGNES).

As técnicas de separação são baseadas em troca iônica (IE) e resinas complexantes (CR), incluindo ainda técnicas de microsseparação, como difusão em filmes finos por gradientes de concentração (DGT), em membranas líquidas suportadas (PLM) e membranas de Donnan (DMT).

Dentre as técnicas eletroanalíticas, tem-se destacado a utilidade de $\mathrm{SCP}^{7}$ e cronopotenciometria de redissolução com varredura de potencial de deposição (SSCP), ${ }^{8}$ sobretudo quando acopladas com a técnica de redissolução em equilíbrio nernstiano na ausência de gradientes (AGNES). ${ }^{9}$ De fato, para SSCP a equação de onda é conhecida rigorosamente no regime de corrente cinética, permitindo assim acesso direto à informação sobre a natureza dinâmica dos complexos metálicos. ${ }^{10,11}$ Isso permitiu criar uma interessante dicotomia entre 
o desenvolvimento experimental destas técnicas e um significativo desenvolvimento teórico, iniciado em 2005 com a publicação de Pinheiro, Van Leeuwen e Minor, ${ }^{12}$ abordando uma nova teoria de especiação dinâmica em dispersões coloidais.

Dentro deste contexto, o presente trabalho tem como objetivo apresentar uma revisão bibliográfica sobre as técnicas de cronopotenciometria de redissolução anódica para estudo da especiação dinâmica de metais traço em ambientes aquáticos, incluindo a interpretação físico-química do sinal obtido em sistemas modelo e meios naturais. Serão apresentadas em maior detalhe algumas aplicações particularmente relevantes, como, por exemplo, estudos de complexação de chumbo e cádmio com substâncias húmicas para ilustrar a heterogeneidade dos ligantes naturais.

\section{Histórico da especiação usando técnicas de redissolução anódica em ambiente aquático}

As técnicas de redissolução são efetuadas em duas etapas: na primeira aplica-se uma diferença de potencial constante no eletrodo de trabalho durante um intervalo de tempo fixo, levando à redução do íon metálico, o qual fica amalgamado no eletrodo de mercúrio ou depositado na superfície de um eletrodo sólido. Em seguida, na etapa de redissolução, o metal amalgamado é reoxidado de modo controlado, de forma a determinar a quantidade de metal reduzido na etapa de deposição.

Na etapa de redissolução é comum usar técnicas voltamétricas como varredura linear (DC-ASV), pulso diferencial (DP-ASV) ou onda quadrada (SW-ASV), sendo o sinal obtido diretamente proporcional à concentração do íon metálico na solução. A característica mais interessante destas técnicas comparada com as voltametrias diretas é a pré-concentração do metal no volume ou superfície do eletrodo (com fatores maiores de 100 e frequentemente maiores que 1000), de modo que a corrente de reoxidação é bem menos perturbada por correntes capacitivas ou impurezas na solução, sendo por isso, normalmente utilizada para análise de soluções extremamente diluídas, da ordem de $10^{-10} \mathrm{~mol} \mathrm{~L}{ }^{-1}$. A descrição matemática do sinal correspondente à reoxidação do metal é complicada e normalmente só existem soluções numéricas para poucos eletrodos de trabalho. ${ }^{13}$

Do ponto de vista analítico as técnicas de redissolução tiveram um desenvolvimento marcante durante a década de 1970, como se pode ler no livro de Vydras et al. ${ }^{14}$ Mas é a partir de 1980, com a crescente comercialização de potenciostatos controlados por computadores pessoais que estas técnicas se generalizaram. ${ }^{15}$

Por outro lado, o desenvolvimento da especiação de metais em ambiente aquático está muito ligado ao livro "Aquatic Chemistry", a obra seminal de Werner Stumm e J. J. Morgan, publicado em 1970 quando Stumm estava em Harvard. ${ }^{16}$ Justamente em 1970, Stumm volta à sua terra natal, a Suíça, mais precisamente Zurique, tornando-se professor titular no Instituto Federal de Tecnologia (ETH) e diretor do EAWAG (Institute for Water Ressources and Water Pollution Control). Neste instituto, que liderou até 1992, ele implementou a sua visão multidisciplinar da pesquisa em ambientes aquáticos, sendo que um dos pilares importantes foi a determinação de metais traço por técnicas eletroquímicas de redissolução. Outros aspectos importantes da sua visão foi a natureza dinâmica dos ambientes naturais, muito impulsionada pelos seus estudos de adsorção em superfícies de partículas minerais, com especial relevância para os oxo-hidróxidos de ferro.

Nas décadas de 1980 e 1990, além dos trabalhos de Werner Stumm no EAWAG, diversos outros grupos e pesquisadores contribuíram de forma relevante para o desenvolvimento dos estudos de especiação de metais traço em ambientes aquáticos, tanto do ponto de vista teórico como do ponto de vista experimental, sendo que dentre estes últimos, as técnicas de redissolução foram importantes devido aos seus baixos limites de detecção. Nesse sentido destacam-se as importantes contribuições dos grupos de Jacques Buffle (CABE, Université de Geneve, Suiça), ${ }^{1}$ Herman Van Leeuwen (University of Wageningen, Holanda), T. M. Florence (CSIRO, Austrália), ${ }^{17}$ Hans Nurnberg e Pavel Valenta (Juelich, Alemanha), ${ }^{18}$ François Morel (University of Princeton, USA) e sua estudante de doutorado Janet Hering (University of North Carolina, USA, recentemente diretora do EAWAG), ${ }^{19}$ Jaume Puy e Josep Galceran (Universidad de Lleida, Espanha).

O contexto do trabalho desenvolvido, especialmente a sua natureza dinâmica, é resumido de forma clara por T.M. Florence ${ }^{20}$ quando diz que a "Anodic stripping voltammetry (ASV) is the technique most commonly used for speciation of heavy metals in waters. It has high sensitivity for the common toxic heavy metals, copper, lead, cadmium and zinc (which are measured in the same analysis), and the ASV-labile metal (ex., the metal measurable by ASV at the natural $\mathrm{pH}$ of the water or in mildly acid conditions) may approximate to the bioavailable fraction".

\section{A natureza dinâmica das técnicas de redissolução}

A questão principal da especiação dinâmica está ligada à biodisponibilidade dos complexos metálicos, ou seja, se estes podem ou não contribuir para a quantidade de metal internalizado por um organismo e, consequentemente, a sua toxicidade.

Neste contexto, em primeiro lugar, deve-se considerar se os complexos metálicos são dinâmicos ou inertes. A natureza dinâmica de um complexo é expressa pela relação entre a cinética da dissociação química e o transporte difusivo (do metal livre e do complexo) na direção de uma interface por efeito do gradiente de concentração. Como esses processos se relacionam a um organismo ou uma técnica analítica, eles são dependentes do tempo de residência do complexo na camada de difusão associada à superficie do organismo ou eletrodo.

Assim, é necessário comparar o tempo característico da técnica (t) (o qual pode ser entendido como o tempo que o íon metálico livre, M, demora para percorrer completamente a camada de difusão), com o tempo de vida característico do íon metálico livre na solução, o que pode ser obtido a partir das constantes de velocidade de associação $\left(\mathrm{k}_{\mathrm{a}}\right.$ ) ou de dissociação $\left(\mathrm{k}_{\mathrm{d}}\right)$, dadas por $1 / \mathrm{k}_{\mathrm{a}} \mathrm{c}_{\mathrm{L}, \mathrm{t}}$ ou $1 / \mathrm{k}_{\mathrm{d}}$, respectivamente, sendo $c_{\mathrm{L}, \mathrm{t}}$ a concentração total do agente complexante L. Para uma escala de tempo $(\mathrm{t})$ muito maior do que o tempo de vida de $\mathrm{M}$ e de seu complexo ML, cada íon metálico individual pode reagir com elevada frequência, passando da condição livre para a forma de complexo no interior da camada de difusão. Esta situação é denominada dinâmica. Quando o tempo de vida de M e ML são maiores do que a escala de tempo operacional, o complexo não se dissocia no interior da camada de difusão. Este é o caso estático, sendo as espécies designadas como complexos inertes, quando os complexos ML não contribuem para o fluxo e apenas os íons metálicos livres podem ser reduzidos no eletrodo. ${ }^{21}$

\section{As reações de associação dos metais traço: o mecanismo de Eigen}

É muito importante referir que o caso da cinética de complexação dos íons metálicos em sistemas aquosos é um caso muito particular na cinética química. De fato, esta segue o mecanismo de Eigen, ${ }^{21,22}$ que consiste em duas etapas principais: (i) formação de um complexo precursor na esfera externa de coordenação (do inglês, outher sphere $(o s)$ ); (ii) posterior liberação da molécula de água da esfera interna (do inglês, inner sphere (is)) com entrada do agente complexante, para formar uma ligação de coordenação com o íon metálico:

$$
\mathrm{M}\left(\mathrm{H}_{2} \mathrm{O}\right)_{6}^{2+}+\mathrm{L}^{-} \underset{k_{d}^{o s}}{\stackrel{k_{a}^{o s}}{\leftrightarrows}}\left\{\mathrm{M}\left(\mathrm{H}_{2} \mathrm{O}\right)_{6} \cdot \mathrm{L}\right\}^{+}
$$




$$
\left\{\mathrm{M}\left(\mathrm{H}_{2} \mathrm{O}\right)_{6} \cdot \mathrm{L}\right\}^{+} \underset{k_{d}^{i s}}{\stackrel{k_{a}^{i s}}{\leftrightarrows}} \mathrm{M}\left(\mathrm{H}_{2} \mathrm{O}\right)_{5} \mathrm{~L}^{+}+\mathrm{H}_{2} \mathrm{O}
$$

em que $k_{a}^{o s}$ e $k_{d}^{o s}$ são, respectivamente, as constantes de velocidade de formação e de taxa de dissociação para o complexo de esfera externa; $\left\{\mathrm{M}\left(\mathrm{H}_{2} \mathrm{O}\right)_{6} \bullet \mathrm{L}\right\}^{+}$, enquanto $k_{a}^{i s}$ e $k_{d}^{i s}$ são as constantes de velocidade para a substituição da molécula de $\mathrm{H}_{2} \mathrm{O}$ por $\mathrm{L}^{-}$na formação do complexo de esfera interna, e vice-versa, respectivamente.

$\mathrm{O}$ aspecto particularmente interessante deste mecanismo é que a etapa limitante da velocidade para a formação do complexo de esfera interna $\left\{\mathrm{M}\left(\mathrm{H}_{2} \mathrm{O}\right)_{6} \bullet \mathrm{L}\right\}^{+}$é a perda de uma molécula de água da esfera interna, sendo, portanto, apenas dependente do íon metálico e logo independente da natureza do ligante.

A constante de velocidade é geralmente nomeada como $\mathrm{k}_{\mathrm{w}}$. Assim, $\mathrm{k}_{\mathrm{a}}^{\text {is }}=\mathrm{k}_{\mathrm{w}}$, sendo o valor de $\mathrm{k}_{\mathrm{w}}$ independente do ligante L. A Tabela 1 mostra alguns valores de $\mathrm{k}_{\mathrm{w}}$ que podem ser encontrados na literatura para diferentes metais. ${ }^{23}$

Tabela 1. Valores de constantes de velocidade de perda da molécula de água da esfera interna de coordenação para alguns metais ${ }^{23}$

\begin{tabular}{ccc}
\hline Íon Metálico & $\boldsymbol{k}_{w} / \mathbf{s}^{-1}$ & Faixa de valores \\
\hline $\mathrm{Pb}^{2+}$ & $7 \times 10^{9}$ & - \\
$\mathrm{Hg}^{2+}$ & $2 \times 10^{9}$ & - \\
$\mathrm{Cu}^{2+}$ & $1 \times 10^{9}$ & $(0,2-5) \times 10^{9}$ \\
$\mathrm{Ca}^{2+}$ & $6 \times 10^{8}$ & $(0,5-9) \times 10^{8}$ \\
$\mathrm{Cd}^{2+}$ & $3 \times 10^{8}$ & $(1-5) \times 10^{8}$ \\
$\mathrm{Zn}^{2+}$ & $7 \times 10^{7}$ & $(3-50) \times 10^{7}$ \\
$\mathrm{Mn}^{2+}$ & $3 \times 10^{7}$ & $(0,4-5) \times 10^{7}$ \\
$\mathrm{Fe}^{2+}$ & $4 \times 10^{6}$ & $(1-4) \times 10^{6}$ \\
$\mathrm{Co}^{2+}$ & $2 \times 10^{6}$ & $(0,2-2) \times 10^{6}$ \\
$\mathrm{Ni}^{2+}$ & $3 \times 10^{4}$ & $(1-4) \times 10^{4}$ \\
$\mathrm{Fe}^{3+}$ & $2 \times 10^{2}$ & $(2-200) \times 10^{2}$ \\
$\mathrm{Al}^{3+}$ & 1 & $0,2-16$ \\
$\mathrm{Cr}^{3+}$ & $5 \times 10^{-7}$ & $(4-6) \times 10^{-7}$ \\
\hline
\end{tabular}

A formação dos complexos de esfera externa é puramente eletroestática. As constantes de velocidade para a formação/dissociação do complexo na esfera externa são oriundas da energia da interação eletrostática adimensional $\left(U^{o s}\right)$ e das mobilidades do $\mathrm{M}\left(\mathrm{H}_{2} \mathrm{O}\right)_{6}^{2+} \mathrm{e}$ $\mathrm{L}^{-}$, representadas por seus respectivos coeficientes de difusão $\left(D_{M} \mathrm{e}\right.$ $D_{L}$ ), conforme apresentado ${ }^{24}$ nas Equações 3 e 4 :

$$
\begin{gathered}
k_{a}^{o s}=4 \pi N_{A V} a\left(D_{M}+D_{L}\right) \frac{U^{o s}}{\exp \left(U^{o s}\right)-1} \\
k_{d}^{o s}=\frac{3\left(D_{M}+D_{L}\right)}{a^{2}} \frac{U^{o s} \exp U^{o s}}{\exp \left(U^{o s}\right)-1}
\end{gathered}
$$

sendo $a$ : distância centro-a-centro de maior aproximação entre o íon complexo $\mathrm{M}\left(\mathrm{H}_{2} \mathrm{O}\right)_{6}^{2+}$ intacto e o ligante $\mathrm{L}^{-}$. Para um único par iônico $M \bullet L$ numa solução eletrolítica, $U^{o s}$ é dado pela Equação 5.

$$
U^{o s}=\left(\frac{z_{M} z_{L} e^{2}}{4 \pi \varepsilon \varepsilon_{0} a k T}\right)\left(1-\frac{\kappa a}{1+\kappa a}\right)
$$

sendo $\varepsilon \varepsilon_{0}$ : permissividade do meio; $z_{M}$ e $z_{L}$ : números algébricos das cargas M e L, respectivamente; $\kappa:$ inverso da distância de Debye.

O primeiro termo entre parêntesis na equação 5 representa a energia primária de Coulomb de atração eletrostática entre o metal e o ligante, e o segundo termo corrige a energia para o efeito de blindagem, devido à presença de eletrólito. Os íons de eletrólito indiferentes na solução diminuem o efeito da atração entre o cátion metálico e o ânion ligante. O parâmetro eletrostático que contabiliza o efeito do eletrólito é a distância de Debye,$^{25}$ geralmente denotado como $\mathrm{K}^{-1}$ :

$$
\kappa^{-1}=\left(\frac{\varepsilon \varepsilon_{0} R T}{F^{2} \sum_{i} z_{i}^{2} c_{i}^{*}}\right)^{1 / 2}
$$

sendo $\sum_{i} z_{i}^{2} c_{i}^{*}$ : soma de todos os íons presentes no meio, representados por sua carga $z_{i}$ e a concentração no interior da solução, $c_{i}^{*}$.

O termo $U^{O S} / \exp \left(U^{O S}\right)$ - 1 na equação 3 se aproxima da unidade quando $U^{O S}$ se aproxima de zero. O limite de zero de $U^{O S}$ corresponde ao processo de encontro dos reagentes $M$ e $L$, unicamente controlado por difusão. A expressão é maior do que 1 para $U^{O S}<0$ (atração), e menor do que 1 para $U^{o s}>0$ (repulsão). A razão $k_{a}^{o s} / k_{d}^{o s}$ é a constante de estabilidade de formação dos complexos de esfera externa, $K^{o S}$, que está relacionada com $U^{O S}$ através da equação: ${ }^{26}$

$$
K^{o s}=\frac{4 \pi N_{A V} a^{3}}{3} \exp \left(-U^{o s}\right)=\frac{\left[\left\{M\left(H_{2} O\right)_{6} \cdot L\right\}^{+}\right]}{\left[M\left(H_{2} O\right)_{6}\right]\left[L^{-}\right]}
$$

No estado estacionário do processo contínuo de formação dos complexos de esfera interna, a concentração do precursor de esfera externa (Equação 1) pode ser considerada como essencialmente independente do tempo. A constante global, $k_{a}$, para a formação do complexo de esfera interna a partir de $\mathrm{M}\left(\mathrm{H}_{2} \mathrm{O}\right)_{6}$ e $\mathrm{L}$-pode ser escrita de acordo com a Equação 8.

$$
k_{a}=\frac{K_{a}^{o s} k_{w}}{K_{d}^{o s}+k_{w}}
$$

Neste contexto, o mecanismo de Eigen apresenta dois casos limitantes: $:^{22,24}$

1. A primeira etapa (Equação 1) é rápida em relação a segunda etapa (Equação 2) e o complexo de esfera externa tende para o estado de equilíbrio com o metal livre $M$. Logo, $k_{a}$ torna-se:

$$
k_{a} \approx K^{o s} k_{w} \text {, pois } k_{d}^{o s} \gg k_{w}
$$

2. Quando a formação do complexo de esfera externa é a etapa limitante da velocidade, tem-se:

$$
k_{a}=k_{a}^{o s} \text {, pois } k_{d}^{o s} \ll k_{w}
$$

Comumente, duas condições podem levar a situações em que os valores de $k_{d}^{o s}$ são semelhantes ou menores que os valores de $\mathrm{K}_{\mathrm{W}}$ : (i) íons metálicos com desidratação muito rápida (valores elevados de $\mathrm{K}_{\mathrm{W}}$ como, por exemplo, $\mathrm{Pb}(\mathrm{II}), \mathrm{Cd}(\mathrm{II}), \mathrm{Hg}(\mathrm{II}), \mathrm{Cu}(\mathrm{II})$ ) e (ii) ligantes com uma carga altamente negativa. A grande negatividade de $\mathrm{z}_{\mathrm{L}}$, leva a um valor correspondentemente grande de atração $U^{O S}$, resultando em um valor relativamente baixo de $k_{a}^{o s}$.

A consequência mais importante do mecanismo de Eigen é que a constante de associação do metal com o ligante depende apenas das características do metal (a sua carga e $\mathrm{K}_{\mathrm{W}}$ ) e da carga do ligante, facilitando muito a interpretação das cinéticas de reação dos metais.

\section{O conceito de labilidade}

Se os complexos são dinâmicos e dissociam na camada de difusão adjacente à superfície do eletrodo ou organismo, ainda temos de considerar o problema do transporte reativo, ou seja, a distinção entre complexos lábeis e não-lábeis. Esta é mais sutil, tendo em vista que ambas definições se referem a sistemas com taxas relativamente altas de conversão de espécies complexadas em íons metálicos livres. 
Os complexos metálicos lábeis são caracterizados por altas velocidades de dissociação/reassociação, nas quais o fluxo de difusão $\left(J_{\text {dif }}\right)$ é muito menor que o fluxo cinético $\left(J_{\text {kin }}\right)$, em qualquer escala espacial relevante, sendo mantido o equilíbrio entre o metal total complexado e livre. Consequentemente, os processos de transporte de metal livre e complexado não podem ser separados, existindo uma difusão acoplada. ${ }^{27}$

Em contraste, os complexos são não-lábeis quando a cinética de dissociação/reassociação é mais lenta, ou seja, em que a taxa de dissociação efetiva é próxima do transporte por difusão (fluxo difusivo menor, ou da mesma ordem de grandeza do fluxo cinético). Nesse caso, a taxa de dissociação (no volume de reação) determina a contribuição do complexo para o fluxo interfacial de íons metálicos. ${ }^{21}$

A distinção entre complexos lábeis, não-lábeis e inertes é de fundamental importância para compreender a especiação dinâmica de metais e depende de vários parâmetros, com especial destaque para o tempo característico do processo, o qual está associado à espessura da camada de difusão. De maneira geral, para uma dada concentração total de metal, a quantidade de metal disponível para participar do processo interfacial (redução ou internalização por um organismo) depende da natureza dinâmica dos complexos em solução. A Figura 1 ilustra esquematicamente os diferentes comportamentos.

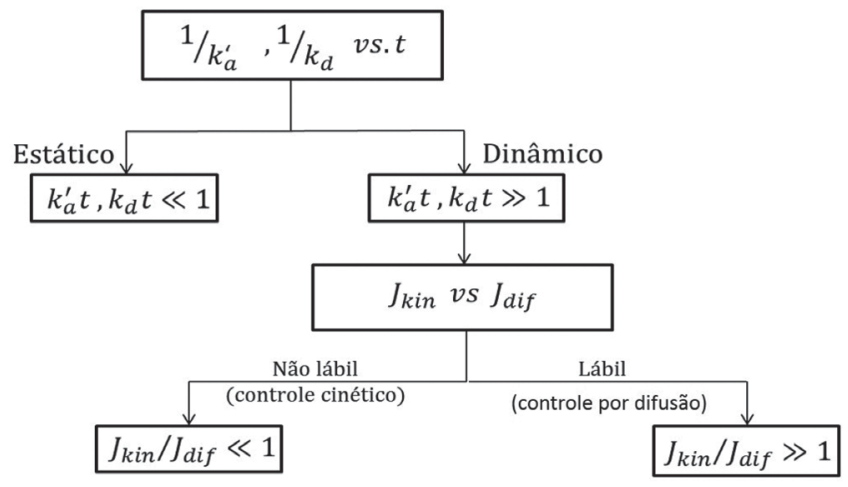

Figura 1. Representação esquemática dos comportamentos característicos de complexos metálicos determinados pela relação entre o intervalo de tempo do experimento, $t$, e a cinética de associação/dissociação do complexo, sendo kin = cinético e dif = difusão. Fonte : adaptado de Mota et al .

No caso de complexos lábeis o sinal das técnicas de redissolução é proporcional à concentração total do metal em solução e no caso de quase-lábeis é proporcional a uma fração do metal total. Então, no caso de sistemas constituídos por complexos quase-lábeis, é praticamente impossível a quantificação correta das espécies em solução por técnicas voltamétricas de redissolução.

Este fato levou, no final da década de 1970, ao aparecimento dos primeiros critérios de labilidade, propostos inicialmente por Davison ${ }^{28}$ para a VRA usando um eletrodo de filme fino de mercúrio (TMFE) e depois por van Leeuwen, ${ }^{29}$ usando o conceito de espessura da camada de reação de Koutecky e Korita. ${ }^{30}$ Os critérios de labilidade são expressos como desigualdades entre o fluxo cinético e o fluxo difusional devido à complexidade das equações que descrevem o sinal experimental. Tais critérios permitem estimar teoricamente se os complexos são lábeis ou não, assegurando a correção dos cálculos de especiação.

\section{As limitações das técnicas de redissolução anódica em ambientes aquáticos naturais}

Além do problema crucial de identificação da natureza dos complexos, as técnicas de redissolução anódica sofrem ainda uma série de problemas técnicos que afetam o seu desempenho em especiação de metais traço em águas naturais.

O principal problema é a adsorção de matéria orgânica presente em águas naturais à superfície do eletrodo devido às suas propriedades hidrofóbicas e/ou a um ganho de entropia. ${ }^{31}$ Em VRA, a adsorção pode estar presente nas etapas de deposição e redissolução, tendo sido apresentada na literatura várias tentativas para discriminar a sua influência em cada uma das etapas. ${ }^{32-34}$ Para diminuir este efeito na etapa de deposição deve se utilizar um potencial de deposição no qual a adsorção de espécies orgânicas seja minimizada. No entanto, vários estudos de isotermas de adsorção de diferentes ácidos húmicos, efetuados para diferentes potenciais aplicados, diferentes $\mathrm{pH}$ e forças iônicas mostram que é impossível evitar totalmente este fenômeno. ${ }^{35-38}$ Outra estratégia é alterar a composição ou mudar a solução da célula na etapa de redissolução, ${ }^{39}$ ou utilizar eletrodos cujas superfícies são modificadas quimicamente para diminuir a adsorção. ${ }^{40-42}$

Outro problema experimental sério em VRA é o conhecido efeito de superfície. Esse efeito decorre do fato de que na etapa de redissolução a concentração de metal nas adjacências do eletrodo se torna muito maior do que no interior da solução, podendo inclusive saturar os sítios de complexação do ligante presente nas mesmas camadas de solução. De fato, para avaliar os parâmetros de complexação a partir destas medidas, a concentração do ligante não complexado na superfície do eletrodo, $c_{L}^{0}$, deve ser a mesma que no interior da solução, $c_{L}$ e a concentração total do ligante, $c_{L, t}$ deve estar em tal excesso que $c_{L}=c_{L, t}$. Para satisfazer esta condição num intervalo de $5 \%$ de erro possível deve-se ter $c_{L, t}>20 c_{M L}^{0}$ para técnicas de redissolução, o que, na presença de uma complexação forte, equivale a $c_{L, t}>20 c_{M, t}^{0}$. Para se respeitar esta condição é necessário que a $c_{L, t}>500 c_{M, t}{ }^{43}{ }^{4}$

Mota et al. ${ }^{44}$ estudaram o efeito da concentração à superfície do eletrodo através da variação de $c_{M, t} / c_{L, t}$, tendo demonstrado que quando $c_{M, t}^{0}$ é menor que $c_{L, t}$ mas não é desprezável frente a este, a altura do pico decresce e a largura à meia altura aumenta comparativamente à ausência de ligante. Tal situação conduz a picos voltamétricos mais baixos e mais largos, ${ }^{45} \mathrm{e}$ o aumento ainda maior de $c_{M t} \cdot t_{d}$ torna o ligante completamente saturado à superfície do eletrodo, dando origem a um ombro no voltamograma.

Quando ocorre a adsorção do ligante no eletrodo, também se observa um efeito de superfície com um comportamento semelhante ao descrito acima, devido à saturação gradual dos sítios de complexação do ligante adsorvido pelo metal. ${ }^{46,47}$

Por outro lado, as técnicas de cronopotenciometria de redissolução anódica, no modo de eletrólise completa na etapa de reoxidação, permitem obter informação dinâmica diretamente e contornar a maioria das limitações das técnicas de VRA. ${ }^{8}$

\section{As técnicas de cronopotenciometria de redissolução anódica}

Nos primeiros trabalhos usando a técnica de cronopotenciometria de redissolução anódica, então denominada "Potentiometric Stripping Analysis" (PSA), ${ }^{48}$ aplicava-se uma corrente de reoxidação relativamente elevada, gerando-se uma difusão linear semi-infinita no interior do eletrodo. Com isso a reoxidação não era quantitativa, de modo que ao final de cada experimento ainda restava metal amalgamado no eletrodo, causando os mesmos inconvenientes observados nas medidas por VRA, como adsorção de compostos orgânicos e efeito de superfície.

Por certo, as técnicas de cronopotenciometria de redissolução anódica apenas são vantajosas quando se aplica uma baixa corrente de reoxidação de tal forma que a reoxidação ocorra lentamente, permitindo que todo o metal seja reoxidado, ou seja, atingindo a depleção completa. Neste caso, temos uma eletrólise para a qual pode-se simplesmente aplicar a lei de Faraday e obter uma relação 
quantitativa direta entre o sinal analítico (tempo de reoxidação) e a concentração do metal na amostra. ${ }^{7}$

A principal vantagem da SCP sobre as técnicas de VRA é a discriminação da carga capacitiva devido à forma de medir o sinal (tempo de reoxidação do metal). Esse tempo é obtido a partir da medida da área do pico obtido da derivada de potencial versus o tempo, eliminando assim todas as correntes capacitivas, incluindo aquelas provocadas pela adsorção. ${ }^{49}$ Além disso, o fato de a SCP ser efetuada no modo de depleção completa faz com que o sinal corresponda ao número de mols de metal amalgamado ou depositado. Medindo-se a área do pico, o sinal deixa de ser perturbado por distorções que afetam as medidas da altura do pico, o que tipicamente ocorre com as técnicas de VRA sujeitas ao efeito de superfície. Ao mesmo tempo, as medidas de SCP necessitam de menor excesso de ligante em solução. ${ }^{50} \mathrm{~A}$ SCP apresenta ainda um limite de detecção comparável com o da voltametria de redissolução por pulso diferencial (DP-ASV), sendo também menos sensível à formação de complexos intermetálicos no amalgama. ${ }^{51}$

Talvez o aspecto mais interessante destas técnicas seja a existência, no caso da SSCP, de uma equação analítica para a onda no regime de corrente cinética. ${ }^{10}$ Foi demonstrado que a partir da curva de SSCP é possivel obter sempre a constante de estabilidade termodinâmica dos complexos, independentemente da natureza dinâmica destes, usando uma expressão do tipo DeFord-Hume. ${ }^{52}$

Além disso, por comparação dos parâmetros característicos da onda da SSCP (o tempo de reoxidação limite e o potencial de deposição de meia-onda, $E_{d, 1 / 2}$ ) se obtém um indicador experimental de labilidade dos complexos metálicos. ${ }^{11}$

Todavia, em presença de ligantes heterogêneos, como as substâncias húmicas, deixa de ser possível interpretar a variação de $E_{d, 1 / 2}$ da SSCP de uma forma simples, pois a heterogeneidade influencia o declive da onda de SSCP. ${ }^{53}$ Nestes casos é aconselhável fazer medidas consecutivas de SSCP e AGNES para obter informação termodinâmica, cinética e sobre a heterogeneidade. ${ }^{54}$ Outras vantagens referem-se ao fato de que utilizando um filme fino de mercúrio as medidas podem conduzidas sem necessidade de desoxigenação prévio da amostra, tornando assim, mais fácil a sua realização in-situ..$^{55}$

A seguir descreve-se em detalhe essas técnicas, os seus fundamentos teóricos, a interpretação do sinal e sua implementação experimental em estudos de especiação em ambientes aquáticos.

\section{Cronopotenciometria de redissolução anódica (SCP)}

Sendo uma técnica de redissolução, a SCP tem a etapa de deposição característica, na qual se aplica um potencial de deposição $\left(E_{d}\right)$ suficientemente negativo para que a concentração de metal livre na superfície do eletrodo tenda a zero durante um intervalo de tempo rigorosamente controlado (tempo de deposição, $t_{d}$ ), normalmente em células eletroquímicas nas quais as condições de transporte de massa do metal do interior da solução para o eletrodo de trabalho são conhecidas. Assim, essa técnica diferencia-se na etapa de reoxidação, na qual a quantificação do metal acumulado é feita por cronopotenciometria, ou seja, aplicando uma corrente de oxidação constante $\left(I_{s}\right)$ e seguindo a variação do potencial do eletrodo de trabalho com o tempo, $E$ versus $t$.

$\mathrm{Na}$ presença de processos faradaicos, o sinal analítico é dado pelo tempo necessário (tempo de reoxidação, $\tau^{*}$ ) para a reoxidação completa do metal depositado. Todavia, é difícil determinar o sinal analítico a partir do cronopotenciograma, pois estas curvas podem ser distorcidas por efeitos de cargas. A forma mais exata é determinar os $\tau^{*}$ a partir da derivada $d t / d E v s E$, visto que a contribuição capacitiva corresponde à região sob a linha de base e a área do pico corresponde unicamente à contribuição faradaica. ${ }^{7}$

Do ponto de vista matemático tem-se nas etapas de deposição e reoxidação:

$$
\begin{aligned}
& \text { n.mol depositado }=I_{d}^{*} t_{d} / n F \\
& \text { n.mol reoxidado }=I_{s} \tau^{*} / n F
\end{aligned}
$$

em que $I_{s}$ é a corrente de reoxidação, $\tau *$ o tempo de reoxidação, $I_{d}{ }^{*}$ a corrente limite de deposição, $t_{d}$ o tempo de deposição, $n$ o número de elétrons envolvido no processo e $F$ a constante de Faraday.

Como no modo da depleção o número de mols de metal depositado é igual ao número de mols de metal reoxidados, então o tempo de transição limite ${ }^{7}$ é dado por:

$$
\tau^{*}=I_{d}^{*} t_{d} / I_{s}
$$

O parâmetro chave aqui é a corrente limite de deposição, $I_{d}{ }^{*}$, que para o caso da redução de um íon metálico na ausência de ligantes é dada por:

$$
I_{d}^{*}=\left(n F A D_{M} c_{M, T}^{*}\right) / \delta_{M}
$$

em que $A$ é a área do eletrodo, $D_{M}$ é o coeficiente de difusão do metal, $c^{*}{ }_{M, T}$ é a concentração total de íon metálico no interior da solução e $\delta_{\mathrm{M}}$ é a espessura da camada de difusão, que depende do tipo de eletrodo de trabalho e das condições hidrodinâmicas da célula eletroquímica.

Os experimentos de SCP têm sido conduzidos principalmente com o eletrodo estático de gota de mercúrio (HMDE) ou com o eletrodo rotativo de filme fino de mercúrio (TMF/RDE). Como os eletrodos de filme fino tem grandes vantagens (uma vez que o $\mathrm{TMF} / \mathrm{RDE}$ tem um volume muito menor e uma área maior do que o HMDE apresenta uma sensibilidade mais elevada) para as técnicas de redissolução, todo o desenvolvimento teórico está relacionado a estes eletrodos. ${ }^{56}$ No caso dos TMF/RDE, a espessura da camada de difusão é dada pela equação de Levitch. ${ }^{57}$

$$
\delta_{M}=1,61 D_{M}^{1 / 3} \omega^{-1 / 2} v^{1 / 6}
$$

em que $\omega$ é velocidade de rotação $\left(\nu_{r o t}\right)$ angular, dada por $\omega=2 \pi v_{r o t}$ e $v$ é o coeficiente de viscosidade da solução em análise.

\section{Interpretação do sinal de SCP do íon metálico em presença de ligantes}

Em matrizes ambientais, é comum considerar a situação de excesso de ligante, isto é, a condição em que a concentração total de ligante é muito superior à concentração total do metal. Assim, o equilíbrio termodinâmico em solução entre o metal e o ligante é descrito pela constante de estabilidade, $K^{\prime}=K c_{L, T}=c_{M L} / c_{M}$, sendo que $c_{L, T}$ representa a concentração total do ligante, $c_{M L}$ a concentração do complexo e $c_{M}$ a concentração de metal livre.

A questão agora é compreender como a presença de ligante, ou seja, a natureza dos complexos formados, vai afetar o sinal de SCP, ou seja, a corrente limite de redução, $I_{d}{ }^{*}$, e o tempo de reoxidação, $\tau *$.

No caso mais simples, isto é, para sistemas estáticos (complexos inertes), todo o metal complexado deixa de estar disponível para ser reduzido e o sinal obtido é diretamente proporcional ao metal livre em solução, $c_{M}{ }^{*}$.

Para os sistemas dinâmicos, a velocidade de dissociação do complexo determina a contribuição do complexo para o fluxo do ín metálico na interface. Este fluxo corresponde à corrente cinética derivada pela aproximação Koutecký e Koryta. ${ }^{30} \mathrm{~A}$ partir da Figura 2, pode-se observar que a camada de difusão é dividida conceitualmente em duas regiões em que os perfis de concentração de $M$ e $M L$ são diferentes.

Na primeira região, de $\delta$ a $\mu$, os perfis são acoplados e, na segunda região, de $\mu$ à superfície do eletrodo, a concentração de $M L$ é 


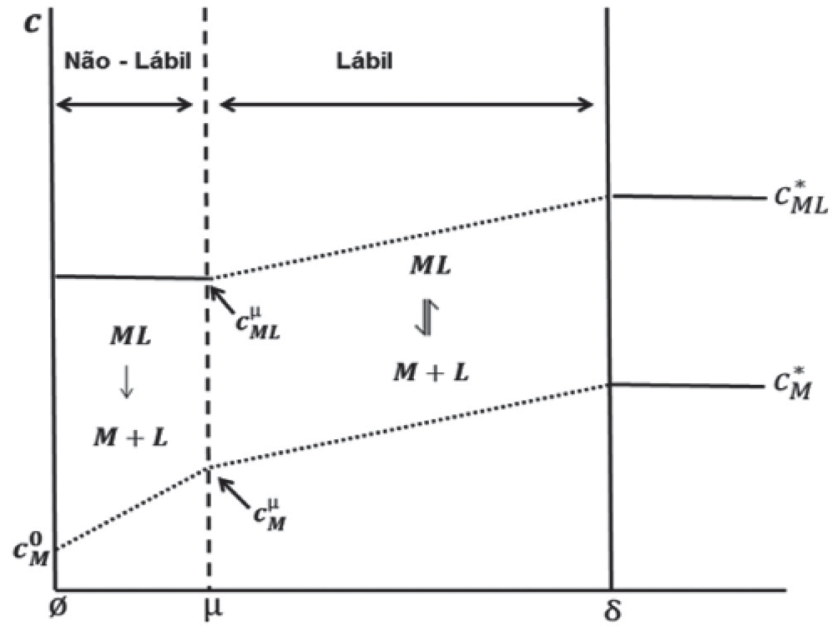

Figura 2. Representação esquemática dos perfis de concentração de $M$ e $M L$, em função da distância, $x$, a partir da superfície do eletrodo $\left(c_{M}^{0}\right)$, ilustrando a camada de reação $\left(c_{M}^{\mu}\right.$ e $\left.c_{M L}^{\mu}\right)$. Fonte: adaptado de Pinheiro et al. ${ }^{62}$

constante, enquanto a de $M$ vai a zero. Estas duas regiões são separadas pelo limite da espessura da camada de reação, $\mu$, que, do ponto de vista físico-químico, corresponde ao percurso do metal livre desde que se dissocia até voltar a se associar, dado por:

$$
\mu=\left(D_{M} / k_{a} c_{L, T}\right)^{1 / 2}
$$

Considera-se que a partir do interior da solução até $\mu$ o equilíbrio entre $\mathrm{M}$ e ML é mantido constante, mas de $\mu$ até à superfície do eletrodo $(\mathrm{x}=0)$ não há equilíbrio, sendo que o fluxo total é dado unicamente pelo fluxo cinético. Esta aproximação é restrita a espessuras de camada de reação menores do que a camada de difusão.

No intervalo de $\mu<x<\delta$ os complexos são lábeis, tendo-se que o fluxo de metal livre e complexado na posição $x=\mu$ é dado pela equação:

$$
J_{\mu}=\bar{D}\left(c_{M, T}^{*}-c_{M, T}^{\mu}\right) /(\bar{\delta}-\mu)
$$

sendo o coeficiente de difusão médio dado por:

$$
\bar{D}=\left(D_{M L} c_{M L}+D_{M} c_{M}\right) /\left(c_{M L}+c_{M}\right)=D_{M}\left(1+\left(D_{M L} / D_{M}\right) K^{\prime}\right) /\left(1+K^{\prime}\right)
$$

e a espessura da camada de difusão média, $\bar{\delta}$, é modificada em conformidade com as condições hidrodinâmicas:

$$
\bar{\delta}=1,61 \bar{D}^{1 / 3} \omega^{-1 / 2} v^{1 / 6}
$$

Por outro lado, entre $0<x<\mu, c_{M L}=c_{M L}^{\mu}$ não há dissociação de complexo, sendo o fluxo à superfície dado por:

$$
J=D_{M}\left(c_{M}^{\mu}-c_{M}^{0}\right) / \mu
$$

Assumindo condições de estado estacionário, é obrigatório que os fluxos a $x=\mu$ e $x=0$ sejam iguais, $J_{\mu}=J$, podendo-se assim resolver a equação 17 para a concentração de metal total no ponto $\mu$ :

$$
c_{M, T}^{\mu}=c_{M, T}^{*}-J(\bar{\delta}-\mu) / \bar{D}
$$

Sabendo-se que neste ponto $(x=\mu)$ o sistema ainda está em equilíbrio, ou seja:

$$
c_{M, T}^{\mu}=c_{M}^{\mu}\left(1+K^{\prime}\right)
$$

a substituição da equação 22 na equação 21 fornece o valor de concentração do íon metálico livre na posição $\mu$ :

$$
c_{M}^{\mu}=\left[c_{M, T}^{*}-J(\bar{\delta}-\mu) / \bar{D}\right] /\left(1+K^{\prime}\right)
$$

Substituindo a equação 23 na equação 20 e a resolvendo em relação ao fluxo, $J$, tem-se:

$$
J=\left(c_{M}^{*}-c_{M}^{0}\right) /\left[(\bar{\delta}-\mu) / \bar{D}\left(1+K^{\prime}\right)+\mu / D_{M}\right]
$$

No caso da corrente limite de deposição em presença de ligantes, ou seja, para potenciais de deposição suficientemente negativos, a concentração de metal na superfície tende para zero, e reconhecendo que o fluxo é $(J=I / n F A)$, obtém-se:

$$
I_{d, M+L}^{*}=n F A c_{M}^{*} /\left[(\bar{\delta}-\mu) / \bar{D}\left(1+K^{\prime}\right)+\mu / D_{M}\right]
$$

Sendo que:

$$
\bar{\delta}=1,61 \bar{D}^{1 / 3} \omega^{-1 / 2} v^{1 / 6}
$$

Assim, para a corrente limite no caso geral dinâmico não é possível discriminar o sinal, pois este depende de três parâmetros: da espessura da camada de reação, $\mu$, da constante de equilíbrio, $K^{\prime}$, e do coeficiente de difusão do complexo, $D_{M L}$, que obviamente não podem ser obtidos a partir de um único sinal experimental.

Por outro lado, no caso de sistemas lábeis, para os quais $\bar{\delta} \gg \mu$, tem-se que $\mu / \mathrm{D}_{\mathrm{M}}$ também é desprezível frente ao primeiro termo, de modo que a equação 25 torna-se:

$$
I_{d, M+L}^{*}=n F A \bar{D}\left(1+K^{\prime}\right) c_{M}^{*} / \bar{\delta}
$$

e como $c_{M, T}=c_{M}(1+K)$ tem-se:

$$
I_{d, M+L}^{*}=n F A \bar{D} c_{M, T}^{*} / \bar{\delta}
$$

Se dividirmos esta equação pela equação 14, ou seja, pela corrente limite obtida na ausência de ligantes, tem-se:

$$
I_{d, M+L}^{*} / I_{d, M}^{*}=(\bar{D} / \bar{\delta}) /\left(D_{M} / \delta_{M}\right)=\left(\bar{D} / D_{M}\right)^{2 / 3}
$$

Se utilizar a equação 13 e resolver para $\bar{D}$, obtem-se:

$$
\bar{D}=D_{M}\left(\tau_{M+L}^{*} / \tau_{M}^{*}\right)^{3 / 2}
$$

equação a partir da qual se pode calcular $K^{\prime}$ conhecendo $D_{M}$ ou $D_{M L}$ e sabendo $K^{\prime}$ (nos casos em que $D_{M L}<D_{M}$ ):

$$
K^{\prime}=\left(D_{M}-\bar{D}\right) /\left(\bar{D}-D_{M L}\right) \text { ou } D_{M L}=\bar{D}-\left(D_{M}-\bar{D}\right) / K^{\prime}
$$

Como se pode observar, a técnica de SCP, tal como as técnicas de VRA, contém muita informação na corrente limite, mas esta não se encontra acessível devido à complexidade do sinal. Nas técnicas de VRA essa limitação é normalmente complementada recorrendo à informação contida na posição do pico no eixo do potencial em presença de ligantes, por comparação à sua posição na ausência destes. Todavia, em SCP esse tipo de análise é muito mais complicada que em VRA, ${ }^{58}$ impossibilitando sua utilização.

Para superar este inconveniente foi introduzida a técnica de SCP por etapas, isto é, a SCP com varredura de potencial de deposição (SSCP, do inglês scanned deposition potential). ${ }^{8}$ Nesta técnica a variação do potencial de meia onda da curva de SSCP, na presença e ausência de ligantes, fornece informação confiável sobre as constantes 
de complexação dos metais ou, no caso de ligantes heterogêneos, sobre o grau de heterogeneidade química dos mesmos. ${ }^{10}$

\section{Cronopotenciometria de redissolução anódica com Varredura de Potencial (SSCP)}

As curvas de SSCP (Figura 3) são construídas a partir de uma série de medidas de SCP individuais obtidas a diferentes potenciais de deposição, $E_{d}$, abrangendo situações em que nenhum metal é reduzido no eletrodo, passando pela região de Nernst, e chegando à região de potenciais onde todo o metal que chega à superfície do eletrodo é reduzido (situação limitada por difusão).

Nesta abordagem, o tempo de reoxidação, , é medido para uma variedade de potenciais de deposição, $E_{d}$, ou seja, a única informação retirada de cada onda individual de SCP é a área do pico.

Em seguida, é apresentada a base teórica descrevendo a curva de SSCP para uma solução contendo apenas íons metálicos ${ }^{8}$ e, posteriormente, para o comportamento completo de corrente cinética, incluindo os respectivos casos limites. Como no caso da SCP, essa descrição será baseada na teoria da camada de difusão no estado estacionário, considerando um eletrodo rotativo de filme fino de mercúrio (TMFE/RDE).

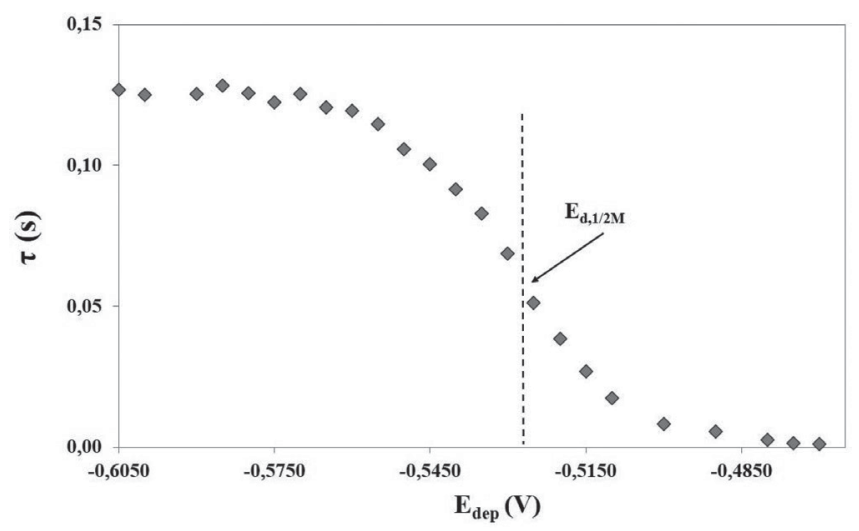

Figura 3. Exemplo de curva de SSCP de Pb(II) $1 \times 10^{-7} \mathrm{~mol} \mathrm{~L}^{-1}$ na ausência de ligante, em $\mathrm{NaNO}_{3} 0,01 \mathrm{~mol} \mathrm{~L} \mathrm{~L}^{-1}$. Experimentos realizados utilizando um eletrodo de filme fino de mercúrio

A redução reversível de um cátion metálico segue um comportamento nernstiano entre as concentrações de metal à superfície do eletrodo de trabalho, em solução $c_{M}^{0}$, e amalgamado no eletrodo, $c_{M^{0}}^{0}$, que pode ser escrito em termos da sua razão, $\theta$ :

$$
\theta=\left(c_{M}^{0} / c_{M^{0}}^{0}\right)=\exp \left(n F\left(E_{d}-E^{0}\right) / R T\right)
$$

Ao aplicar uma baixa corrente de reoxidação, ou seja, no modo de depleção completa, estamos na situação em que a concentração de metal reduzido à superfície do eletrodo, $c_{M^{0}}^{0}$, e no interior do eletrodo, $c_{M^{0}}^{*}$, são idênticas, ou seja, não é necessário considerar um gradiente de concentração ou fluxo no interior do eletrodo.

Durante o tempo de deposição a concentração de metal amalgamado aumenta até atingir $c_{M^{0}}^{*}$ ao tempo $t_{d}$, o que pode ser representado pela integral no tempo da corrente de deposição, $I_{d}$ :

$$
c_{M^{0}}^{*}=\int I_{d} d t /(n F V)
$$

em que V é o volume do eletrodo.

Ao mesmo tempo, à superfície do eletrodo, mas do lado da solução, $c_{M}^{0}$, pode ser obtida a partir da corrente de redução:

$$
I_{d}=n F A D_{M}\left(c_{M}^{*}-c_{M}^{0}\right) / \delta_{M}
$$

Substituindo-se as equações 31 e 32 na expressão de Nernst (Equação 30), obtém-se:

$$
\theta=\exp \left(n F\left(E_{d}-E^{0}\right) / R T\right)=\left(c_{M}^{*}-I_{d} \delta_{M} /\left(n F A D_{M}\right)\right) /\left(\int I_{d} d t /(n F V)\right)
$$

A solução desta equação integral simples em função da corrente é:

$$
I_{d}(t)=I_{d}^{*} \exp \left(-t / \tau_{d}\right)
$$

em que $\tau_{\mathrm{d}}$ é a constante de tempo característico do processo de deposição definida por:

$$
\tau_{d}=V \delta_{M} /\left(A D_{M} \theta\right)
$$

A carga total, $Q$, depositada no eletrodo durante a etapa de deposição, $t_{d}$, pode ser obtida por integração da equação 34 entre $t=0$ e $t_{d}$ :

$$
Q=I_{d}^{*} \tau_{d}\left[1-\exp \left(-t_{d} / \tau_{d}\right)\right]
$$

Como em SCP a carga obtida na etapa de deposição tem que ser igual à carga medida na etapa de redissolução $\left(Q=I_{S} \tau\right)$, a equação da curva de SSCP, que descreve a relação entre $\tau$ e $E_{d}$ no regime de depleção completa, é:

$$
\tau=\left(I_{d}^{*} \tau_{d} / I_{s}\right)\left[1-\exp \left(-t_{d} / \tau_{d}\right)\right]
$$

O valor limite do tempo de reoxidação $\left(\tau^{*}\right)$, é obtido para os potenciais de deposição muito maiores do que o potencial padrão $\left(E_{d}>E_{0}\right)$, ou seja, quando $\theta \rightarrow 0$. Neste caso $\tau_{d}$ torna-se muito maior que $t_{d}\left(t_{d} / \tau_{d}<<1\right)$, e o termo exponencial da equação 37 pode ser desenvolvido como uma série, na qual se desprezam os termos de ordem superior resultando na equação 13:

$$
\tau^{*}=\left(I_{d}^{*} \tau_{d} / I_{s}\right)\left[1-\left(1-\frac{t_{d}}{\tau_{d}} \ldots . .\right)\right]=\left(I_{d}^{*} t_{d} / I_{s}\right)
$$

\section{Interpretação do sinal de SSCP para o íon metálico em presença de ligantes}

Em presença de excesso de ligantes formando complexos 1:1 com o íon metálico, a equação de Nernst pode ser escrita em função do metal total presente em solução como:

$$
\theta=\left(c_{M, t}^{0} / c_{M^{0}}^{0}\right) /\left(1+K^{\prime}\right)
$$

No caso geral a equação da curva de SSCP é dada a partir da Equação 37, sendo o $I_{\mathrm{d}}^{*}$ substituído por $I_{\mathrm{d}, \mathrm{M}+\mathrm{L}}^{*}$ (Equação 25):

$$
\tau=\left(I_{d, M+L}^{*} \tau_{d, M+L} / I_{s}\right)\left[1-\exp \left(-t_{d} / \tau_{d, M+L}\right)\right]
$$

e na equação 35 em presença de ligantes o termo, $\tau_{d, M+L}$, será dado por:

$$
\tau_{d, M+L}=V \bar{\delta} /\left(A \bar{D}\left(1+K^{\prime}\right) \theta\right)
$$

A curva de SSCP na região da corrente limite em presença de ligantes é equivalente ao sinal de SCP e pode ser analisada conforme explicado anteriormente. Assim, o interesse adicional da SSCP corresponde à região onde se observa o deslocamento do potencial devido à formação de complexos. Para equações de onda polarográfica esta análise é feita recorrendo à equação de DeFord-Hume ${ }^{52}$ que relaciona a variação dos potenciais de meia-onda, $\Delta E_{d, 1 / 2}$, com a constante de estabilidade dos complexos, $K^{\prime}$. No caso da SSCP foi demonstrado que apesar de forma da curva não ser idêntica à de uma 
curva polarográfica, a equação de DeFord-Hume é válida, mesmo para sistemas não lábeis: ${ }^{11}$

$$
E_{1 / 2, \mathrm{M}+\mathrm{L}}-E_{1 / 2, \mathrm{M}}=-\frac{R T}{n F} \ln \left(1+K^{\prime}\right)-\frac{R T}{n F} \ln \left(\tau_{\mathrm{M}+\mathrm{L}}^{*} / \tau_{\mathrm{M}}^{*}\right)
$$

em que o termo experimental $\tau_{\mathrm{M}+\mathrm{L}}^{*} / \tau_{\mathrm{M}}^{*}$ é apenas uma correção geométrica relacionada à diminuição da corrente limite devido à perda de labilidade e/ou à diminuição do coeficiente de difusão no caso de ligantes macromoleculares, ou seja, em sistemas onde $D_{M L}<D_{M}$.

A Figura 4 exemplifica duas curvas de SSCP, uma em que só existe metal $(M)$ e outra em presença de ligantes complexantes $(M+L)$, evidenciando os dois resultados experimentais independentes: as variações do potencial de meia onda e do tempo de transição limite.

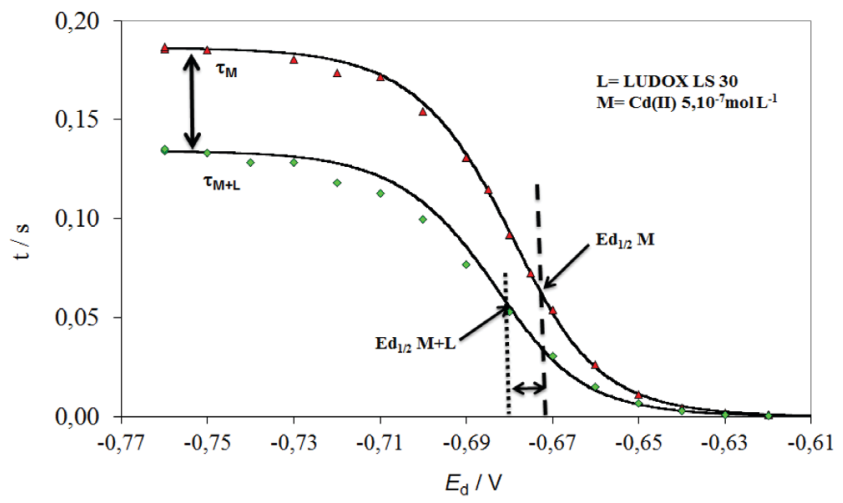

Figura 4. Exemplo de curva de SSCP na ( $\mathbf{\Delta})$ ausência do ligante, $M, e(\diamond) n a$ presença do ligante, $M+L$, indicando a variação do potencial de deposição, $E_{d, 1 / 2}$ e tempo de transição limite, $\tau$. Condições: FI: 0,01 mol $L^{-1}, \mathrm{pH} 7,5$, concentração de Cd(II): $5,04 \times 10^{-7} \mathrm{~mol} \mathrm{~L}^{-1}$, razão $\mathrm{M} / \mathrm{L} 1: 1$

Um dos aspectos mais relevantes da SSCP é que o tempo de transição sempre reflete a magnitude do fluxo da deposição original, independentemente da sua natureza (ou seja, controlada por difusão ou controlada cineticamente), de modo que a constante $K^{\prime}$ pode ser calculada a partir desta, independentemente do grau de labilidade do sistema. ${ }^{11}$

Se a adsorção de substâncias orgânicas, o excesso de superfície e a natureza dinâmica dos complexos não afetam os resultados obtidos pela equação de DeFord-Hume em SSCP, o mesmo já não se pode dizer da heterogeneidade química dos complexos em solução.

Em sistemas heterogêneos, o sinal de SSCP torna-se mais difícil de analisar, tornando-se necessário complementar esta técnica com outras para compreender a especiação, tornando AGNES extremamente interessante, ${ }^{59}$ pois permite determinar o íon metálico livre usando o mesmo sistema experimental e com limites de detecção similares.

\section{Técnica de redissolução nernstiana na ausência de gradientes (AGNES)}

A redissolução nernstiana na ausência de gradientes (AGNES) é uma técnica eletroquímica de redissolução utilizada para a determinação direta da concentração de metal livre. ${ }^{9} \mathrm{Na}$ etapa de deposição se aplica um potencial próximo ao potencial de redução padrão do metal até o momento em que o equilíbrio termodinâmico entre o metal amalgamado e o interior da solução seja atingido.

A concentração acumulada depende apenas da concentração de íon metálico no interior da solução $\left(c_{M}^{*}\right)$ e é independente de qualquer outra característica do meio, como a complexação ou adsorção eletrolítica. No fim da etapa de deposição, obtém-se a razão entre o metal amalgamado e em solução, segundo a Equação de Nernst:

$$
Y=\frac{c_{\mathrm{M}, \mathrm{amalg}}}{c_{\mathrm{M}}^{*}}=\exp \left[\frac{-n F\left(E_{\mathrm{d}}-E^{0}\right)}{R T}\right]
$$

em que Y: representa o ganho (ou fator de pré-concentração); $c_{\mathrm{M} \text {,amalg: }}$ : concentração de íon metálico amalgamado; $E^{0}$ : potencial de redução padrão do metal; $E_{\mathrm{d}}$ : potencial de deposição.

$\mathrm{Na}$ etapa de reoxidação pretende-se apenas quantificar o metal amalgamado, tendo sido usadas várias técnicas diferentes para isso. Ao fazer sequencialmente AGNES e SSCP a forma mais fácil de quantificar o metal amalgamado em AGNES é usar uma etapa de reoxidação similar à $\mathrm{SCP} .{ }^{60} \mathrm{Na}$ segunda etapa o tempo de reoxidação

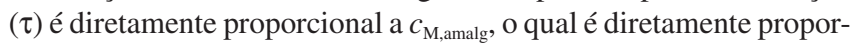
cional à concentração de íon metálico no interior da solução segundo:

$$
\tau=Y c_{M}^{*}
$$

Assim, a AGNES funciona de forma análoga a um eletrodo de íons seletivo (EIS), permitindo a determinação direta do valor do íon metálico livre na solução, com limites de detecção muito baixos, que, por exemplo, são da ordem de nanomolar para Cd(II) com tempos de deposição de 1 a 2 minutos em eletrodos de filme fino de $\mathrm{Hg} .{ }^{61}$ Além disso, conhecendo o metal livre na solução e o balanço de massa para o metal, o valor da constante de estabilidade $K$ é obtido diretamente.

\section{COMO ESTUDAR A ESPECIAÇÃO DINÂMICA DE METAIS TRAÇO USANDO TECNICAS CRONOPOTENCIOMÉTRICAS}

Neste momento é importante explicar como se usam estas técnicas para estudar a especiação dinâmica com os diferentes tipos de ligantes possíveis, pois dependendo de suas características e do conhecimento que temos deles, a quantidade de experimentos necessários pode variar bastante.

Note que, tanto para a SSCP como para AGNES, uma calibração prévia é sempre necessária.

A onda de SSCP produz duas informações experimentais distintas:

i) o tempo de reoxidação limite $\left(\tau_{M+L}^{*}\right)$, que depende da constante de estabilidade termodinâmica, $K^{\prime}\left(K^{\prime}=K^{\prime} c_{L, T}\right)$, do coeficiente de difusão do complexo, $D_{M L}$, e da constante de velocidade de associação, $k_{a}^{\prime}$ (através da espessura da camada de reação, $\mu$ ),

ii) a variação dos potenciais de onda, sendo o mais importante o de meia-onda, $\Delta E_{\mathrm{d}, 1 / 2}$, que em sistemas homogêneos só depende da constante de estabilidade termodinâmica, $K^{\prime}$, e que em sistemas quimicamente heterogêneos depende também do grau de heterogeneidade.

Em sistemas complexos deve-se complementar com AGNES para obter a concentração de metal livre no interior da solução, $\mathrm{c}_{\mathrm{M}}^{*}$.

Em relação aos tipos de ligantes, de uma forma relativamente simples podemos identificá-los como:

Tipo 1) ligantes pequenos contendo dois ou três grupos funcionais conhecidos, respeitando a condição $D_{M}=D_{M L}$;

Tipo 2) macromoléculas ou nanopartículas quimicamente homogêneas, mas de dimensão maior que o íon metálico, apresentando $D_{M L}<<D_{M}$;

Tipo 3) macromoléculas (substâncias húmicas, partículas minerais, etc.) ou nanopartículas quimicamente heterogêneas, $D_{M L}<<D_{M}$;

Tipo 4) misturas de ligantes, de um ou vários tipos anteriores (por exemplo: mistura de ligantes presentes em águas naturais)

Relembrando que em estudos de complexação dinâmica estamos fundamentalmente interessados na natureza dos complexos, os quais 
podem ser complexos inertes, que não dissociam na espessura da camada de difusão e não contribuem para o sinal, complexos lábeis, que estão em equilíbrio em todos os pontos da camada de difusão e podem ser completamente reduzidos durante o tempo de deposição, e complexos quase-lábeis, dos quais apenas uma fração pode ser reduzida durante o tempo de deposição. Relembre-se, ainda, que a natureza dinâmica de um complexo depende da escala de tempo da técnica, que por sua vez depende da espessura da camada de difusão.

A Figura 5 demonstra os sinais de SSCP obtidos para os ligantes pequenos (tipo $1, D_{M}=D_{M L}$ ). A característica dos complexos inertes (Figura 5a) é que como apenas o íon metálico é reduzido, o sinal apresenta o mesmo potencial de meia-onda do metal livre. Por outro lado, o valor de tempo de reoxidação limite $\left(\tau_{M+L}^{*}\right)$ corresponde apenas ao metal livre e $\operatorname{logo}$ diminui de acordo com o fator $c_{M} / c_{M, T}$. Este comportamento é característico dos complexos inertes, qualquer que seja o tipo de ligante, pois em qualquer caso apenas o íon metálico é reduzido, e logo, se efetuarmos AGNES, devemos obter exatamente o mesmo valor.

No caso dos complexos lábeis (Figura $5 \mathrm{~b}$ ), o valor de $\tau_{M+L}^{*}$ é igual ao valor obtido na calibração, pois todo o íon metálico é reduzido, mas a presença de complexos desloca o potencial de meia-onda e podemos obter o valor de $K^{\prime}$ a partir da equação 39 .

Para os complexos quase-lábeis (Figura 5c) temos um deslocamento de potencial de meia-onda devido à presença de complexos, a partir do qual se pode obter igualmente o valor de $K^{\prime}$ (equação 39). Além disso, observa-se um decréscimo do valor de $\tau_{M+L}^{*}$, que neste caso é exclusivamente provocado pela perda de labilidade (pois $D_{M}=D_{M L}$ ), a partir do qual se pode obter o valor de $\mu$ (equação 25 ) e, logo, o valor de $k_{a}^{\prime}$ (equação 16).

No caso dos ligantes de tipo 2 (macromoléculas ou nanopartículas quimicamente homogêneos), como o $D_{M L}<D_{M}$, o valor de $\tau_{M+L}^{*}$ será menor que o valor da calibração, quer para o caso lábil, quer para o caso quase-lábil.

A abordagem clássica numa dispersão coloidal ou sistema nanoparticulado quimicamente homogêneo é estudar o efeito do $\mathrm{pH}$ e da força iônica na especiação dinâmica.

No caso de complexos lábeis (Figura 4), a variação do potencial de meia-onda, $\Delta E_{\mathrm{d}, 1 / 2}$, fornece a constante de estabilidade, $K^{\prime}$. Como o decréscimo de $\tau_{M+L}^{*}$ depende somente de $K^{\prime}$ e $D_{M L}$ (equação 31 ), pode-se também usar esta técnica para obter o coeficiente de difusão dos ligantes. ${ }^{62}$

No caso quase-lábil o decréscimo de $\tau_{M+L}^{*}$ depende de $K^{\prime}, D_{M L}$ e $\mu$ (equação 25), mas $\Delta E_{\mathrm{d}, 1 / 2}$ continua dependendo somente da constante de estabilidade, $K^{\prime}$. Nesse caso, temos uma das potencialidades mais interessantes da SSCP, que é a do diagnóstico de labilidade incorporado nos resultados experimentais. De fato, desde que o valor de $D_{M L}$ seja conhecido, podemos calcular $K^{\prime}$ a partir do decréscimo de $\tau_{M+L}^{*}$ como se o sistema fosse lábil (equação 31) e comparar com o valor de $K^{\prime}$ obtido através de $\Delta E_{\mathrm{d}, 1 / 2}$ (equação 39). $\mathrm{Se}$ os valores forem iguais, o sistema é labil, mas se o valor do decréscimo de $\tau_{\mathrm{M}+\mathrm{L}}^{*}$ for maior, então pode-se inferir que o sistema está perdendo labilidade.

Podemos ver um exemplo na Figura 6, em que a variação do pH induz a desprotonação dos ligantes na superfície das partículas de látex carboxiladas, ${ }^{11}$ o que induz um aumento da concentração de ligante desprotonado $\left(c_{L, T}\right) \mathrm{e}$, logo, uma perda de labilidade, expressa pelo afastamento dos pontos experimentais da simulação das curvas de SSCP lábeis usando os valores de $K^{\prime}$ da equação 39.

Note-se que a observação direta da perda de labilidade não nos permite sempre obter um valor confiável de constante de associação, $k_{\mathrm{a}}$, devido aos erros experimentais associados às medidas e à propagação de erro neste cálculo, a qual é aumentada pela raiz quadrada da equação 16.
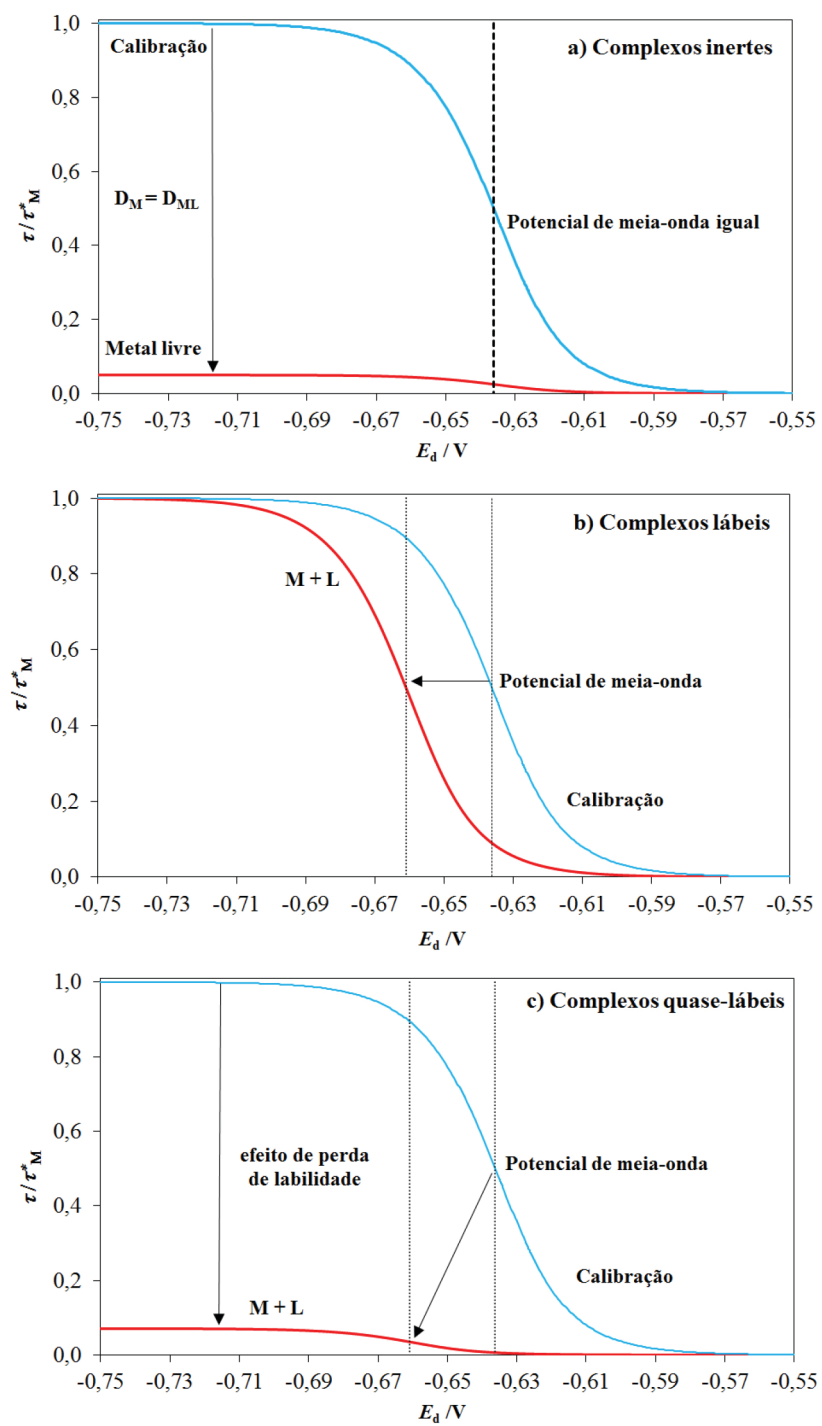

Figura 5. Exemplo genérico de curva SSCP para Cd(II) normalizada pela calibração (linha azul), e na presença do ligante (linha vermelha), $M+L$, indicando (a) a situação de complexos inertes; (b) a situação de complexos lábeis e (c) a situação de complexos quase-lábeis

No caso dos ligantes de tipo 3 (macromoléculas ou nanopartículas quimicamente heterogêneas), que é o caso mais comum em sistemas naturais, com participação predominante da matéria orgânica natural $(\mathrm{MON})$, constituída principalmente pelas substâncias húmicas, a distinção entre complexos lábeis e quase-lábeis é dificultada pela heterogeneidade do sistema.

A abordagem clássica é tentar criar um modelo eletrostático, caracterizando o sistema por titulações ácido-base em meios de diferentes forças iônicas, para depois investigar a especiação dinâmica através de titulações com o íon metálico de interesse, de forma a obter informação sobre a heterogeneidade química utilizando uma vasta faixa de razões metal/ligante em diferentes $\mathrm{pH}$.

Nestes casos, é muito importante efetuar também a quantificação de metal livre por AGNES.

A concentração do metal livre obtida por AGNES permite, em conjunto com o balanço de massa, calcular o valor de $K^{\prime}$. A comparação deste com o $K^{\prime}$ obtido por $\Delta E_{\mathrm{d}, 1 / 2}$ permite aferir se o sistema é quimicamente heterogêneo, e comparando com o $K^{\prime}$ obtido pelo decréscimo de $\tau_{M+L}^{*}$, verificar se o sistema é lábil.

O problema nestes sistemas macromoleculares é obter os valores de $D_{M L}$, pois alguns desses sistemas são polidispersos. Para se 


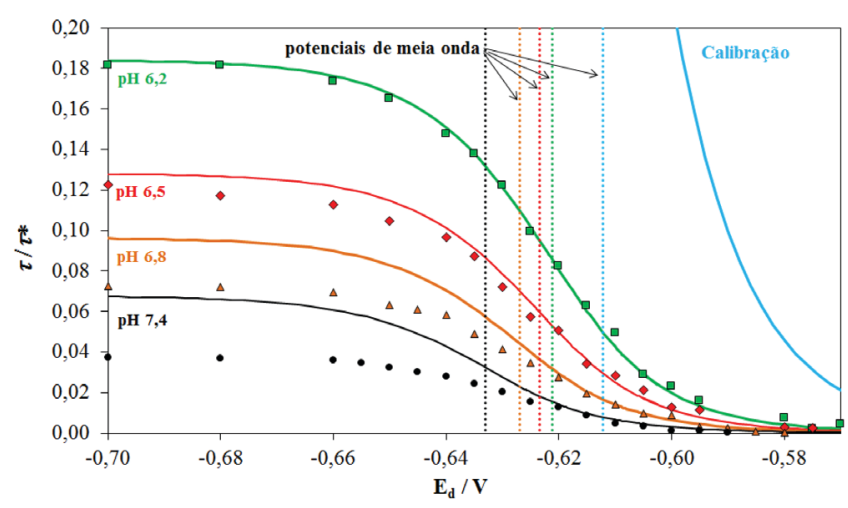

Figura 6. Dados experimentais de SSCP e respectivas simulações para o caso labil de um sistema de Cd(II)/nanopartículas de látex carboxiladas em diferentes valores de $\mathrm{pH}$. As curvas experimentais foram obtidas com solução de $\mathrm{Cd}(\mathrm{II}) 2 \times 10^{-4} \mathrm{~mol} \mathrm{~m}^{-3}$ em $\mathrm{KNO}_{3} 0,1 \mathrm{~mol} \mathrm{~m}^{-3}$, a diferentes $\mathrm{pH}$. Dados Pinheiro e Van Leeuwen ${ }^{11}$

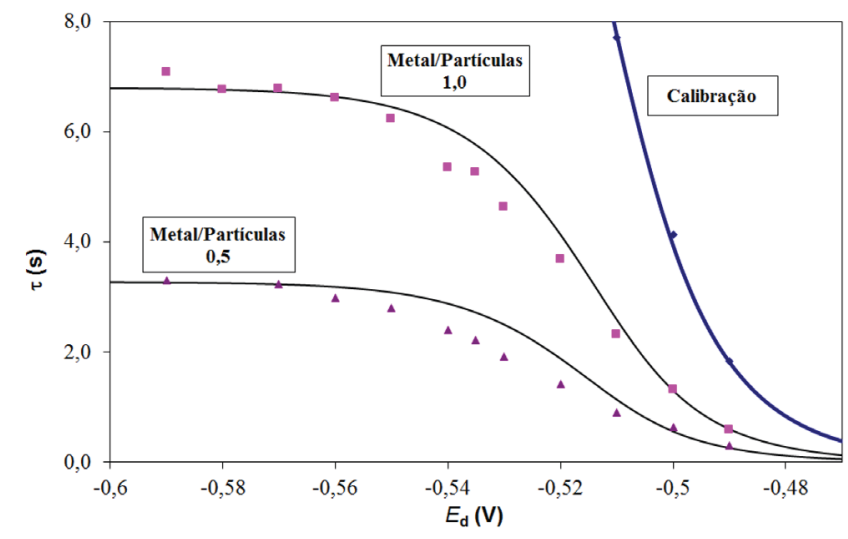

Figura 7. Dados experimentais de SSCP e respectivas simulações para o caso labil homogêneo para um sistema de Pb(II)/nanopartículas de silica a diferentes razões metal/partículas. Dados: Goveia et al. ${ }^{64}$

contornar essa dificuldade, podem-se realizar medidas alternativas, como a espectroscopia de correlação de fluorescência, ou as próprias medidas de SSCP/AGNES usando complexos metálicos tendencialmente lábeis e relativamente pouco heterogêneos, como ocorre de maneira geral com complexos de $\mathrm{Cd}(\mathrm{II}){ }^{62}$

Uma vez que a curva de SSCP é constituída por um conjunto de medidas individuais obtidas a diferentes potenciais de deposição, e que para cada um destes existe na superfície do eletrodo uma relação metal/ligante diferente no caso de sistemas heterogêneos, cada ponto da curva de SSCP corresponderá a um valor de constante de equilíbrio diferente, podendo ainda haver perda de labilidade com este aumento da constante de estabilidade. ${ }^{63}$ Assim, o efeito da heterogeneidade na curva SSCP é alterar a inclinação na zona nernstiana, como se pode observar na Figura 7. Conforme se progride para potenciais mais negativos de $E_{d}$, observa-se um desvio dos pontos experimentais (sistema heterogêneo) em relação à curva simulada (sistema homogêneo). ${ }^{64}$

$\mathrm{O}$ fenômeno da heterogeneidade da complexação de cátions metálicos com substâncias húmicas tem sido objeto de vários modelos, como o modelo da função de equilíbrio diferencial (DEF), que se baseia numa isoterma de Freundlich proposto por Buffle et al.,${ }^{65} \mathrm{e}$ os modelos do tipo NICA propostos por Van Riemsdijk et al. ${ }^{66}$

A curva de SSCP contém uma quantidade de informação sobre heterogeneidade equivalente a uma titulação cobrindo várias ordens de grandeza da razão metal/ligante. No entanto, a extração dessa informação não é simples. Vários estudos de heterogeneidade têm sido realizados sobre o sinal de SSCP com diferentes níveis de sucesso, começando por modelos do tipo isoterma de Freundlich (DEF) ${ }^{53,63,67}$ e pela análise completa da onda de SSCP proposta por Serrano et al. ${ }^{68}$

Os resultados têm sido muito influenciados pelos erros experimentais, aumentados pela natureza logarítmica das equações e pela complexidade das expressões matemáticas envolvidas. Atualmente este é um dos tópicos mais interessantes a serem desenvolvidos.

Ligantes de tipo 4, ou seja, misturas de ligantes de um ou vários dos tipos anteriores, que se encontram normalmente em águas naturais, são difíceis de estudar diretamente. A principal questão está relacionada com a interpretação do sinal, pois estas misturas são polidispersas e polifuncionais. Neste caso é importante caracterizar o máximo possível a amostra, sobretudo a natureza dos ligantes (MON, partículas minerais, outros ligantes orgânicos), bem como as condições ambientais de pH e salinidade, além da concentração total de metal presente.

Os maiores desafios neste momento são os estudos de sistemas ternários compostos por misturas de cátions metálicos, matéria orgânica natural (MON) e partículas minerais (oxohidróxidos de ferro, argilominerais e partículas contendo cálcio, como calcita e apatita), sobretudo pela tendência da MON em adsorver à superficie das partículas, processo cuja cinética é mais lenta que a da complexação dos metais com ambos. Em comparação com os sistemas binários (íon metálico/ligante) existem muito poucos estudos de sistemas ternários.

Nestas matrizes é importante utilizar AGNES para obter a concentração de metal livre e compreender o sinal de SSCP para inferir a informação dinâmica e da heterogeneidade da mistura. Num sistema complexo, a interpretação do sinal vai, por exemplo, requerer o conhecimento dos coeficientes de difusão dos ligantes majoritários, como demonstraram Rocha et al. ${ }^{69}$ num estudo de misturas de polímeros de diferentes tamanhos.

Outra possibilidade é fracionar a amostra por tamanho, por exemplo, usando ultrafiltração, e estudar as frações, ${ }^{70}$ sendo importante realizar estudos com várias técnicas para caracterizar e compreender as interações entre os diferentes componentes da matriz. Ocasionalmente os íons metálicos podem ser usados como sondas para comprender outros processos.

\section{ESTUDOS DE ESPECIAÇÃO DINÂMICA DE METAIS TRAÇO EM SISTEMAS MODELO E MEIOS NATURAIS}

\section{As principais linhas do desenvolvimento teórico da especiação dinâmica}

A publicação de uma equação de onda analítica para o regime de corrente cinética da curva de $\mathrm{SSCP}^{10} \mathrm{em} 2004$, permitindo acesso direto à informação sobre a natureza dinâmica dos complexos metálicos, iniciou uma era de desenvolvimento teórico sobre a cinética dos processos de complexação de metais traço com partículas naturais e antrópicas, iniciado em 2005 com a publicação de uma nova teoria de especiação dinâmica dos metais traço em dispersões coloidais por Pinheiro, van Leeuwen e Minor. ${ }^{12}$

De uma forma breve pode-se referir que inicialmente as teorias de especiação dinâmica foram desenvolvidas supondo que a distribuição dos ligantes era homogênea em relação ao volume da solução, desconsiderando a natureza coloidal de uma grande parte dos ligantes presentes em águas naturais. Esta nova teoria ${ }^{12}$ assume que os ligantes estão localizados dentro da geometria das partículas coloidais de superfície e demonstrou que os valores das constantes de formação/dissociação encontrados para estes sistemas podem ser muito diferentes daqueles encontrados para uma solução verdadeiramente homogênea.

A principal diferença é que o transporte local controlado por difusão de íons metálicos para a partícula deve ser levado em consideração 
pois pode alterar significativamente a constante de associação efetiva, denominada $k_{a}^{* \prime}$, quando comparada com a constante de associação do mecanismo de Eigen, $K_{a}$, que é utilizada no caso de uma solução homogênea. ${ }^{12}$ Esta nova constante pode ser expressa por:

$$
k_{a}^{* \prime}=k_{a}^{\prime} /\left(1+k_{a}^{\prime}\left(4 \pi a D_{M} c_{p}\right)^{-1}\right)
$$

em que $c_{\mathrm{p}}$ é a densidade do número de partículas e $a$ é o raio da partícula.

Essa constante de associação efetiva, $k_{a}^{* \prime}$, apresenta dois valores limites: ${ }^{12}$

(1) o limite difusivo para $k_{a}^{\prime}\left(4 \pi a D_{M} c_{p}\right)^{-1} \gg 1$, em que $k_{a}^{* \prime}=4 \pi a D_{M} c_{p}$. Essa situação ocorre somente para metais que tenham valores de $k_{w}$ relativamente altos;

(2) o limite cinético para $k_{a}^{\prime}\left(4 \pi a D_{M} c_{p}\right)^{-1} \gg 1$, em que $k_{a}^{* \prime}=k_{a}^{\prime}$, esperado para metais que possuem baixos valores de $k_{w}$; em que encontramos de novo a constante de associação de Eigen.

A amplitude da diferença entre $k_{\mathrm{a}}$ e $k_{\mathrm{a}}{ }^{*}$ pode ser de várias ordens de grandeza, de modo que os complexos com ligantes coloidais se tornam menos dinâmicos ou mesmo estáticos para partículas de maiores dimensões. Note-se que no limite difusivo, como os coeficientes de difusão dos diferentes cátions metálicos $\left(D_{M}\right)$ são muito semelhantes, $k_{\mathrm{a}}{ }^{*}$ vai depender do tamanho $(a)$ e concentração $\left(c_{\mathrm{p}}\right)$ das partículas, ou seja, torna-se independente do metal.

Em seguida, o mesmo grupo investigou a labilidade em sistemas eletroquímicos com eletrodos planares, ${ }^{71}$ desenvolvendo um critério de labilidade em sistemas com transporte por convecção. ${ }^{72}$ Foi concluído que num sistema particulado, com os ligantes localizados na superfície da partícula, a desprotonação dos ligantes em função da variação do $\mathrm{pH}$ faz variar a labilidade do sistema mais rapidamente que o aumento do número de partículas.

Em 2008, Duval et al. ${ }^{73}$ expandiram essa teoria para sistemas em suspensões de partículas coloidais permeáveis (géis) e semi-permeáveis (core-shell), onde os sítios de ligação são distribuídos de acordo com perfis espaciais tridimensionais. Em 2009 foi adicionado o efeito da dupla camada elétrica do ponto de vista teórico, ${ }^{74}$ mais tarde verificado experimentalmente. ${ }^{75}$

Uma evolução interessante foi o estudo da labilidade de complexos em presença de misturas de ligantes. Salvador e $_{\text {al. }}{ }^{76}$ propuseram teoricamente que ao adicionar um ligante que forma complexos mais lábeis a um sistema, a labilidade dos complexos já presentes nesse sistema aumenta e, ao adicionar um ligante que forma complexos menos lábeis, a labilidade dos complexos já presentes em solução diminui devido ao acoplamento de todos os processos de associação/ dissociação na matriz. Este efeito foi verificado experimentalmente em 2010 por SCP e AGNES em duas matrizes: Cd/NTA/glicina e $\mathrm{Cd} / \mathrm{NTA}$ /ácido citrico. ${ }^{77}$

Em 2011, van Leeuwen et l. $^{78}$ apresentaram uma teoria para partículas esféricas permeáveis com distribuição homogênea de ligantes e cargas, seguida em $2013^{79}$ de uma interessante publicação sobre a extraordinária reatividade de nanopartículas. Neste trabalho se explica que, para nanopartículas carregadas, a reatividade se deve fundamentalmente a uma acumulação do tipo de Boltzmann de reagentes iônicos e a uma aceleração condutiva do seu transporte. Em seguida, esses conceitos foram generalizados para toda a dinâmica de complexação de íons metálicos com nanopartículas, incluindo o papel central do transporte por condução-difusão, a partição dos íons entre a solução e o volume da partícula e as dinâmicas de associação/ dissociação com os sítios reativos dentro do volume da partícula, ${ }^{80}$ tendo extendido esta aproximação para distribuições heterogêneas de ligantes no volume da partícula em $2014 .{ }^{81}$

Em 2017, Town et al. ${ }^{82}$ questionam a definição da espessura da camada de reação num sistema coloidal sugerindo que a cinética intrapartícula afeta a reatividade na superfície do eletrodo. No seguimento deste trabalho, Duval e colaboradores redefiniram o conceito de camada de reação para o caso de sistemas coloidais ${ }^{83}$ e apresentaram as diferentes situações possíveis para o caso de partículas rígidas, semipermeáveis (core-shell) e permeáveis (géis) ${ }^{84}$

Em termos de trabalhos de revisão podemos citar que em 2009 van Leeuwen e Buffle ${ }^{85}$ apresentaram uma revisão detalhada dos princípios das cinéticas de complexação de ions metálicos com agentes complexantes de diferentes tamanhos. Em 2012, Mota et $a l .{ }^{86}$ reportaram os principais desenvolvimentos teóricos na interpretação das características cinéticas da interconversão de espécies de complexos metálicos considerando três aspectos distintos: o efeito de protonação do ligante, a ligação iônica com macromoléculas fortemente carregadas (por exemplo, os ácidos fúlvicos) e a introdução da teoria dinâmica para a ligação de metal em dispersões coloidais. ${ }^{12}$

Finalmente, em 2017, van Leeuwen et al. ${ }^{87}$ apresentaram uma revisão crítica sobre a cinética de associação de cátions com diferentes tipos de nanopartículas, identificando a contribuição do campo elétrico criado pelas cargas da partícula e identificando os fatores que determinam qual a etapa limitante do processo global de associação/ dissociação.

\section{Exemplos de estudos de especiação dinâmica em sistemas modelo e naturais}

Desde 2004 surgem numerosos trabalhos utilizando o excelente potencial das técnicas de SCP, SSCP e AGNES para estudar a especiação de metais tanto em sistemas modelos quanto em sistemas naturais. Algumas revisões críticas foram publicadas sobre $\mathrm{SCP}{ }^{88}$ comparando-a com outras técnicas não-eletroquímicas ${ }^{89,90}$ ou com outras técnicas eletroanalíticas. ${ }^{86,91}$

Todavia, o número de estudos em que o foco é a especiação dinâmica é menor, devido à maior dificuldade da interpretação desses resultados e ao menor número de modelos teóricos adequados a descrevê-los. Uma boa parte destes estudos tem sido realizada para comprovar as teorias que abordamos no tópico anterior, não sendo necessariamente repetida aqui. Neste tópico será descrito de forma sucinta algumas das publicações mais interessantes como ilustração do potencial destas técnicas e da metodologia para estudar alguns dos problemas de especiação dinâmica relevantes atualmente.

Em 2007, van Leeuwen e Town ${ }^{92}$ estudaram por SSCP as correntes cinéticas de redução de complexos $M L_{2}$ e concluiram que a etapa limitante era a dissociação de $M L \rightarrow M$. A metodologia usada para estudar este processo pode ser aplicada a quaisquer complexos de ordem $\mathrm{ML}_{\mathrm{n}}$.

Domingos et al..$^{93}$ usaram os resultados de SSCP para aferir a capacidade da técnica de permeação em membranas líquidas (PLM) de realizar estudos de especiação dinâmica. Os estudos efetuados usando partículas de diferentes tamanhos permitiram concluir que a análise do sinal de PLM era muito complicada, pois com o aumento de tamanho da partícula o controle do transporte do íon metálico através da membrana se alterava.

Rotureau e van Leeuwen ${ }^{94,95}$ estudaram a cinética da complexação de $\mathrm{Pb}$ (II) com carboximetildextranas, mostrando que a labilidade dos complexos evolui com o tempo, numa escala de várias horas, começando por serem lábeis e tornando-se progressivamente menos lábeis, concluindo que os íons $\mathrm{Pb}$ (II) provocam uma reorganização estrutural no ligante. Estes trabalhos demonstraram que os íons metálicos podem ser usados como sondas para estudar o comportamento dos ligantes, neste caso evidenciando uma reorganização estrutural numa macromolécula.

Em 2011, Goveia et al. ${ }^{64}$ estudaram a complexação de $\mathrm{Pb}$ (II) com nanopartículas de sílica (Ludox LS30, d=7,5 nm), tendo verificado que 
em pH 6,5 e força iônica $1,5 \mathrm{mmol} \mathrm{L}^{-1}$ os complexos eram heterogêneos e perdiam labilidade em concentrações elevadas de partículas. Este sistema tem um interesse particular, pois a pequena dimensão das partículas e a excelente sensibilidade da SSCP permitiram estudar sistemas onde o número de partículas é superior ao número de íons metálicos em solução.

Em 2012, Town et al. ${ }^{96}$ estudaram a interação Cu(II) e Ni(II) com substâncias húmicas usando as novas teorias dinâmicas e observaram que para o íon cobre, ${ }^{96} \mathrm{com}$ a diminuição da força iônica, o mecanismo de difusão pode influenciar a cinética, mesmo para moléculas de dimensões não muito grandes como as substâncias húmicas. Num segundo estudo, verificaram que para o íon cuja desidratação é lenta, como o níquel, o mecanismo de Eigen continua válido, mas para íons cuja desidratação é rápida, como o $\mathrm{Cu}\left(\mathrm{H}_{2} \mathrm{O}\right)_{6}{ }^{2+}$, a aceleração do transporte por condução prevê constantes cinéticas de dissociação pelo menos uma ordem de magnitude maiores para força iônicas entre $0,01-0,1 \mathrm{~mol} \mathrm{dm}^{-3}$, podendo ser ainda maiores nas forças iônicas típicas de águas doces. ${ }^{97}$

Rotureau, ${ }^{98}$ em 2014, realizou um estudo de interação de Cd(II) e $\mathrm{Pb}$ (II) com partículas de argila, mostrando que os complexos de $\mathrm{Cd}(\mathrm{II})$ são homogêneos e lábeis e os de $\mathrm{Pb}$ (II) são heterogêneos e também lábeis. Neste trabalho, os coeficientes de difusão das partículas foram determinados a partir dos estudos com Cd(II), demonstrando mais uma vez a possibilidade de usar estas técnicas para estudar propriedades dos ligantes.

Diaz de Alba et al. ${ }^{99}$ estudaram a especiação de $\mathrm{Pb}(\mathrm{II})$ em água do mar sintética e natural com um conteúdo de $2 \mathrm{mg} \mathrm{L}^{-1}$ de matéria orgânica natural (NOM) usando SSCP e AGNES em diferentes valores de pH entre 2,7 e 8,6. Os resultados mostram complexos lábeis abaixo de $\mathrm{pH}$ 7,0 e uma acentuada perda de labilidade acima deste valor.

Em 2015, Domingos et al. ${ }^{100}$ estudaram a influência das camadas poliméricas de policarboxilatos que recobrem nanopartículas de $\mathrm{CdTe/CdS} \mathrm{na} \mathrm{estabilização} \mathrm{da} \mathrm{nanopartícula} \mathrm{e} \mathrm{na} \mathrm{interação} \mathrm{com} \mathrm{outros}$ cátions metálicos presentes no meio ambiente. Os experimentos usando SSCP mostraram que apesar da solubilidade das nanopartículas ser maior a $\mathrm{pH} 8,5$ do que a $\mathrm{pH}$ 6,0, a quantidade de $\mathrm{Cd}$ (II) livre em solução era muito menor, pois este está complexado na camada polimérica, e que em presença de $\mathrm{Pb}$ (II), este podia tanto ser complexado pela camada, como trocar com o $\mathrm{Cd}$ (II) e formar $\mathrm{PbS}$ na superficie da partícula. Este estudo mostra a versatilidade da SSCP como técnica para estudar o destino de nanopartículas metálicas no meio ambiente.

Town ${ }^{101}$ investigou a competição entre $\mathrm{Ca}$ (II) e $\mathrm{Pb}$ (II) na complexação com substâncias húmicas por SSCP, tendo observado um grande aumento de labilidade dos complexos de $\mathrm{Pb}$ por adição daquele.

Rotureau et al. ${ }^{102}$ investigaram o efeito da temperatura sobre a labilidade dos sitemas Cd-NTA e Pb-IDA usando SSCP, tendo verificado que a labilidade aumenta com a temperatura devido ao aumento da constante de velocidade de associação e da espessura da camada de difusão.

Em 2016, Rotureau et al. ${ }^{103}$ utilizaram Cd(II) como sonda para estudar, por SSCP, os efeitos estruturais em duas partículas core-shell de polimetil metacrilato/N-isopropilacrilamida (PMMA/PNIPAM) carboxiladas com ácidos acrílico ou metacrílico multireativas. Estas partículas sofrem alterações estruturais por efeito da temperatura, $\mathrm{pH}$ e força iônica, sendo que tais alterações são refletidas por variações da heterogeneidade das constantes de equilíbrio com Cd(II).

Vale a pena ressaltar que os experimentos utilizando AGNES, SCP e SSCP para os estudos em sistemas modelo e/ou naturais foram desenvolvidos originalmente com a aplicação em eletrodos de mercúrio, principalmente com o eletrodo estático de gota de mercúrio $(\mathrm{HMDE})^{104-107}$ ou com o eletrodo rotativo de filme fino de mercúrio (TMF/RDE). 8,28,29,55,56,57,61,108 Embora os eletrodos de filme de mercúrio ofereçam alta sensibilidade, a busca por materiais alternativos ${ }^{109}$ que apresentem desempenho eletroquímico e resultados semelhantes surgiu devido aos problemas associados com a utilização e manipulação desse metal, tais como toxicidade, volatilidade e dificuldade para descarte adequado. ${ }^{110,111}$ Nesse sentido, foi demonstrado que eletrodos de filme de Bi podem ser usados para determinação de $\mathrm{Pb}$ (II) livre por AGNES na presença de vários ligantes. ${ }^{109}$ Nos últimos anos, os eletrodos impressos têm sido uma alternativa interessante, apresentando como vantagens: baixo custo de produção, facilidade de modificação para aumento de seletividade e/ou sensibilidade, podendo ainda ser usados em medidas in situ (devido ao seu pequeno tamanho), além de minimizar problemas de contaminação. ${ }^{108,112-115}$ Sensores impressos de ouro, por exemplo, foram usados para investigar as interações dinâmicas de $\mathrm{Hg}$ (II) com ácidos húmicos e suspensões de microalgas. ${ }^{110,111}$

Assim, estudos recentes têm demosntrado que metais como ouro e bismuto apresentam propriedades eletroquímicas que permitem seu uso em especiação dinâmica, sendo considerados "ambientalmente amigáveis" por serem menos tóxicos e terem enorme potencial para viabilizar a realização de estudos on site. ${ }^{109-111,116}$

\section{CONCLUSÕES}

Neste artigo abordamos os desenvolvimentos teóricos e metodológicos das técnicas cronopotenciométricas no estudo da especiação dinâmica de metais traço. Estas técnicas permitiram desenvolver a determinação de constantes de estabilidade de complexos de metais traço e o estudo das suas características dinâmicas com ligantes de ocorrência naturais e antrópicos em ambientes aquáticos, abrindo caminho ao desenvolvimento de novas teorias físico-químicas de especiação dinâmica em sistemas coloidais e novas possibilidades de estudo dos ligantes macromoleculares ou particulados utilizando íons metálicos como sondas. Dentre as novas possibilidades, estamos desenvolvendo uma metodologia de AGNES/SSCP para a determinação do $\mathrm{Hg}$ (II) livre e dos seus parâmetros dinâmicos na presença de diferentes ligantes orgânicos, com o uso de um sistema automatizado por análise por injeção sequencial (SIA, sequential injection analysis). Além disso, em termos de novas aplicações, pode-se investigar a especiação dinâmica com materiais usados para tratamento de água, tais como microalgas, sorventes sintéticos (polímeros com grupo funcionais complexantes) e/ou naturais (argilominerais).

\section{REFERÊNCIAS}

1. Buffle, J.; Complexation reactions in aquatic systems: An analytical approach, Ellis Horwood: Chichester, 1988.

2. Templeton, D. M.; Freek, A.; Cornelis, R.; Danielssom, L. C.; Muntau, H.; van Leeuwen, H. P.; Lobinski, R.; Pure Appl. Chem. 2000, 72, 1470.

3. Buffle, J.; Wilkinson, K. J.; van Leeuwen, H. P.; Environ. Sci. Technol. 2009, 43, 7174 .

4. Buffle, J.; Parthasarathy, N.; Djane, N. K.; Matthiasson, L. Em IUPAC Series Permeation Liquid Membranes for Field Analysis and Speciation of Trace Compounds in Waters in In Situ Monitoring of Aquatic Systems Chemical Analysis and Speciation; Buffle, J., Horvai, G., eds.; Chichester, 2000b, cap 10.

5. Sigg, L.; Black, F.; Buffle, J.; Cao, J.; Cleven, R.; Davison, W.; Galceran, J.; Gunkel, P.; Kalis, E.; Kistler, D.; Martin, M.; Noel, S.; Nur, Y.; Odzak, N.; Puy, J.; van Riemsdijk, W.; Temminghoff, E.; TercierWaeber, M. L.; Toepperwien, S.; Town, R. M.; Unsworth, E.; Warnken, K. W.; Weng, L. P.; Xue, H. B.; Zhang, H.; Environ. Sci. Technol. 2006, 40, 1941.

6. Pesavento, M.; Alberti, G.; Biesuz, R.; Anal. Chim. Acta 2009, 631, 141.

7. Town, R. M.; van Leeuwen, H. P.; J. Electroanal. Chem. 2001, 509, 65.

8. van Leeuwen, H. P.; Town, R. M.; Environ. Sci. Technol. 2003, 37, 3952. 
9. Galceran, J.; Companys, E.; Puy, J.; Cecilia, J.; Garces, J. L. J.; J. Electroanal. Chem. 2004, 566, 109.

10. Van Leeuwen, H. P.; Town, R. M.; J. Electroanal. Chem. 2004, 561, 74.

11. Pinheiro, J. P.; van Leeuwen, H. P.; J. Electroanal. Chem. 2004, 570, 75.

12. Pinheiro, J. P.; Minor, M.; van Leeuwen, H. P.; Langmuir 2005, 21, 8642.

13. Bard A. J.; Faulkner L. R.; Electrochemical Methods: Principles and Applications, 2n $2^{\text {nd }}$ ed., Wiley: New York, 2001.

14. Vydra, F.; Stulik, K.; Julakova, E.; Electrochemical Stripping Analysis, Halstead: New York, 1977.

15. Wang, J.; Stripping Analysis: Principles, Instrumentation and Applications, VCH: Michigan, 1985.

16. Stumm, W.; Morgan, J. J.; Aquatic Chemistry, Wiley: New York, 1970.

17. Florence, T. M.; Analyst, 1986, 111, 489.

18. Nürnberg, H. W.; Valenta, P.; Em Proceedings of NATO Advanced Research Institute on Trace Metals in Sea Water; Wong, C. S., Boyle, E., Bruland, K. W., Burton, J. D., Goldberg, E. D., eds.; Sicily, 1981.

19. Morel, F. M. M.; Hering, J. G.; Principles and applications of aquatic chemistry, Wiley: New York, 1993.

20. Florence, T. M.; Talanta 1982, 29, 364.

21. van Leeuwen, H. P.; Electroanalysis 2001, 13, 830.

22. Eigen, M.; Pure Appl. Chem. 1963, 6, 97.

23. Margerum, D. W.; Cayley, G. R, Weatherburn, D. C. Pagenkopf, G. K. Em Coordination Chemistry; Martell, A.; ed.; Monograph American Chemical Society: Washington, D. C., 1978.

24. van Leeuwen, H. P.; Town, R. M.; Buffle, J.; Langmuir 2011, 27, 4519.

25. Debye, P.; Trans. Electrochem. Soc. 1942, 82, 272.

26. Fuoss, R. M.; J. Am. Chem. Soc. 1958, 80, 5061.

27. van Leeuwen, H. P.; Buffle, J.; Lovric, M.; Pure Appl. Chem. 1992, 64, 1028.

28. Davison, W.; J. Electroanal. Chem. 1978, 87, 395.

29. van Leeuwen, H. P.; J. Electroanal. Chem. 1979, 99, 93.

30. Koutecky, J.; Korita, J.; Electrochim. Acta 1961, 3, 318.

31. Mota, A. M.; Santos, C. M. M. Em Trace metal speciation of labile chemical species in natural waters: Electrochemical methods. In Metal speciation and bioavailability; Tessier, A., Turner, D., eds.; John Wiley \& Sons: New York, 1995, cap.5.

32. Ugapo, T.; Pickering, W. F.; Talanta 1985, 32, 131.

33. Gregor, J. E.; Powel, H. K. J.; Anal. Chim. Acta 1988, 211, 141.

34. Plavsic, M.; Cosovic, B.; Miletic, S.; Anal. Chim. Acta 1991, 255, 15.

35. Raspor, B.; Nurnberg, H. W.; Valenta, P.; Branica, M.; Mar. Chem. 1984, 15, 217.

36. Raspor, B.; Sci. Total Environ. 1989, 81, 319.

37. Mota, A. M.; Pinheiro, J. P.; Gonçalves, M. L. S.; Water Res. 1994, 28 , 1296.

38. Pinheiro, J. P.; Mota, A. M.; Gonçalves, M. L. S.; van Leeuwen, H. P.; Environ. Sci. Technol. 1994, 28, 2119.

39. Morrison, G. M. P.; Florence, T. M.; Stauber, J. L.; Electroanalysis 1990, 2, 9 .

40. Murray, R. M.; Em Electroanalytical Chemistry: A Series of Advances; Bard, A. J., ed.; Marcel Dekker: New York, 1984.

41. Ryan, M. D.; Chambers, J. Q.; Anal. Chem. 1992, 64, 116.

42. Rocha, L. S.; Pinheiro, J. P. Carapuça, H. M.; Langmuir 2006, $22,8247$.

43. Buffle, J.; J. Electroanal. Chem. 1981, 125, 273.

44. Mota, A. M.; Buffle, J.; Kounaves, S. P.; Gonçalves, M. L. S.; Anal. Chim. Acta. 1985, 172, 30.

45. Filella, M.; Buffle, J.; van Leeuwen, H. P.; Anal. Chim. Acta. 1990, 232, 223.

46. Buffle, J.; Vuileumier, J. J.; Tercier, M. L.; Parthasarathy, N.; Sci. Total Environ. 1987, 60, 96.

47. Bugarin, M. G.; Mota, A. M.; Pinheiro, J. P.; Gonçalves, M. L. S.; Anal. Chim. Acta. 1994, 294, 281.

48. Jagner, D.; Analyst 1982, 107, 593.
49. Town, R. M.; van Leeuwen, H. P.; J. Electroanal. Chem. 2002, 523, 15. 50. van Leeuwen, H. P.; Town, R. M.; J. Electroanal. Chem. 2002, 523, 25.

51. Town, R. M.; van Leeuwen, H. P.; J. Electroanal. Chem. 2004, 573, 157.

52. De Ford, D. D.; Hume, D. N.; J. Am. Chem. Soc. 1951, 73, 5321.

53. Town, R. M.; van Leeuwen, H. P.; Aust. J. Chem. 2004, 57, 992.

54. Domingos, R.F.; Huidobro, C.; Companys, E.; Puy, J; Galceran, J.; Pinheiro, J. P.; J. Electroanal. Chem. 2008, 617, 148.

55. Parat, C.; Schneider, A.; Castetbon, A.; Potin-Gautier, M.; Anal. Chim. Acta 2011, 688, 162.

56. Rocha, L. S.; Pinheiro, J. P.; Carapuça, H. M.; J. Electroanal. Chem. 2007, 610, 45.

57. Levich,V. G. Physicochemical Hydrodynamics, Prentice-Hall: New Jersey, 1962.

58. Town, R. M.; van Leeuwen, H. P.; J. Electroanal. Chem. 2002, 535, 25.

59. Domingos, R. F.; Huidobro, C.; Companys, E.; Puy, J.; Galceran, J.; Pinheiro, J. P.; J. Electroanal. Chem. 2008, 617, 148.

60. Parat, C.; Authier, L.; Aguilar, D.; Companys, E.; Puy, J.; Galceran, J.; Potin-Gautier, M.; Analyst 2011, 136, 4337.

61. Rocha, L. S.; Companys, E.; Galceran, J.; Carapuca, H. M.; Pinheiro, J. P.; Talanta 2010, 80, 1887.

62. Pinheiro, J. P.; Domingos, R. F.; Lopez, R.; Brayner, R.; Fievet, F.; Wilkinson, K.; Colloids Surf., A 2007, 295, 200.

63. Town, R. M.; Environ. Sci. Technol. 2008, 42, 4014.

64. Goveia, D.; Pinheiro, J. P.; Milkova, V.; Rosa, A. H.; van Leeuwen, H. P.; Langmuir 2011, 27, 7877.

65. Buffle, J.; Altmann, R. S.; Filella, M.; Tessier, A.; Geochim. Cosmochim. Acta. 1990, 54, 1535.

66. Kinniburgh, D. G.; van Riemsdijk, W. H; Koopal, L. K; Borkovec, M.; Benedetti, M. F; Avena, M. J.; Colloids Surf., A 1999, 151, 166.

67. Domingos, R. F.; Lopez, R.; Pinheiro, J. P.; Environ. Chem. 2008, 5, 24.

68. Serrano, N.; Diaz-Cruz, J. M.; Arino, C.; Esteban, M.; Puy, J.; Companys, E.; Galceran, J.; Cecilia, J.; J. Electroanal. Chem. 2007, 600, 275.

69. Rocha, L. S.; Botero, W. G.; Alves, N. G.; Moreira, J. A.; Da Costa, A. M. R.; Pinheiro, J. P.; Electrochim. Acta. 2015, 166, 402.

70. Monteiro, A. S. C.; Parat, C.; Rosa, A. H.; Pinheiro, J. P.; Talanta 2016, $152,118$.

71. Pinheiro, J. P.; Minor, M.; van Leeuwen, H. P.; J. Electroanal. Chem. 2006, 587, 292

72. Pinheiro, J. P.; Domingos, R. F.; Minor, M.; van Leeuwen, H. P.; J. Electroanal. Chem. 2006, 596, 64.

73. Duval, J. F. L.; Pinheiro, J. P.; van Leeuwen, H. P.; J. Phys. Chem., A 2008, 112, 7137.

74. Duval, J. F. L.; J. Phys. Chem. A 2009, 113, 2275.

75. Duval, J. F. L.; Farinha, J. P. S.; Pinheiro, J. P.; Langmuir 2013, 29, 13835.

76. Salvador, J.; Garces, J. L.; Galceran, J.; Puy, J.; J. Phys. Chem. B 2006 , $110,13661$.

77. Pinheiro, J. P.; Salvador, J.; Companys, E.; Galceran, J.; Puy, J.; Phys. Chem. Chem. Phys. 2010, 12, 1131.

78. van Leeuwen, H. P.; Town, R. M.; Buffle, J.; Langmuir 2011, 27, 4514.

79. van Leeuwen, H. P.; Buffle, J.; Duval, J. F. L.; Town, R. M.; Langmuir 2013, 29, 10302.

80. Town, R. M.; Buffle, J.; Duval; J. F. L.; van Leeuwen, H. P.; J. Phys. Chem. A 2013, 117, 7654.

81. Polyakov, P. D.; Duval, J. F. L.; Phys. Chem. Chem. Phys. 2014, 16, 2010.

82. Town, R. M.; Pinheiro, J. P.; van Leeuwen, H. P.; Langmuir 2017, 33, 527.

83. Duval, J. F. L.; Town, R. M.; van Leeuwen, H. P.; J. Phys. Chem. C. 2017, 121, 19147.

84. Duval, J. F. L.; Phys. Chem. Chem. Phys. 2017, 19, 11815.

85. van Leeuwen, H. P.; Buffle, J. ; Environ. Sci. Technol. 2009, 43, 7175. 
86. Mota, A. M.; Pinheiro, J. P.; Goncalves, M. L. S.; J. Phys. Chem. A 2012, 116, 6442 .

87. van Leeuwen, H. P.; Duval, J. F. L.; Pinheiro, J. P.; Blust, R.; Town, R. M.; Environ. Sci.: Nano 2017, 4, 2133.

88. Serrano, N; Diaz-Cruz, J. M.; Arino, C.; Esteban, M.; Electroanalysis 2007, 19, 2049.

89. Sigg, L.; Black, F.; Buffle, J.; Cao, J.; Cleven, R.; Davison, W.; Galceran, J.; Gunkel, P.; Kalis, E.; Kistler, D.; Town, R. M.; van Leeuwen, H. P.; Environ. Sci. Technol. 2006, 40, 1941.

90. Pesavento, M.; Alberti, G.; Biesuz, R.; Anal. Chim. Acta 2009, 631, 141.

91. Companys, E.; Galceran, J.; Pinheiro, J. P.; Puy, J.; Salaün, P.; Curr. Opin. Electrochem. 2017, 3, 144.

92. van Leeuwen, H. P.; Town, R. M.; J. Electroanal. Chem. 2006, 587, 154.

93. Domingos, R. F.; Benedetti, M. F.; Pinheiro, J. P.; Anal. Chim. Acta. 2007, 589, 261.

94. Rotureau, E.; van Leeuwen, H. P.; J. Phys. Chem. A 2008, 112, 7177.

95. Rotureau, E.; van Leeuwen, H. P.; J. Phys. Chem. A 2009, 113, 12879.

96. Town, R. M.; Duval; J. F. L.; Buffle, J.; van Leeuwen, H. P.; J. Phys. Chem. A 2012, 116, 6489.

97. Town, R. M.; van Leeuwen, H. P.; Buffle, J.; Environ. Sci. Technol. 2012, 46, 10498.

98. Rotureau, E.; Colloids Surf., A 2014, 441, 291.

99. Diaz, A. M.; Galindo R. M. D.; Pinheiro, J. P.; Environ. Chem. 2014, 11, 149.

100. Domingos, R. F.; Franco, C.; Pinheiro, J. P.; Environ. Sci. Pollut. Res. 2015, 22, 2900.

101. Town, R. M.; Environ. Chem. 2015, 12, 137.

102. Rotureau, E.; Waldvogel, Y.; Present, R. M.; Pinheiro, J. P.; J. Electroanal. Chem. 2015, 752, 68.
103. Rotureau, E.; Waldvogel, Y.; Pinheiro, J. P.; Farinha, J. P. S.; Bihannic, I.; Present, R. M.; Duval, J. F. L.; Phys. Chem. Chem. Phys. 2016, 18, 31711.

104. Galceran, J.; Companys, E.; Braungardt, C.; Worsfold, P.; Puy, J.; Anal. Chim. Acta 2016, 917, 40.

105. Chito, D.; Weng, L.; Galceran, J.; Companys, E.; Puy, J.; van Riemsdijk, W. H.; van Leeuwen, H. P.; Sci. Total Environ. 2012, 421-422, 244.

106. Aguilar, D.; Parat, C.; Galceran, J.; Companys, E.; Puy, J.; Authier, L.; Potin-Gautier, M.; J. Electroanal. Chem. 2013, 689, 283.

107. Galceran, J.; David, L. C.; Companys, E.; Castro, C. R.; Salvador, J.; Puy, J.; J. Electroanal. Chem. 2014, 722-723, 118.

108. Rocha, L. S.; Companys, E.; Carapuça, H. M.; Pinheiro, J. P.; Talanta 2010, $80,1887$.

109. Rocha, L. S.; Galceran, J.; Puy, J.; Pinheiro, J. P.; Anal. Chem. 2015, 87, 6078.

110. Do Nascimento, F. H.; Masini, J. C.; Talanta 2012, 100, 57.

111. Do Nascimento, F. H.; Rigobello-Masini, M.; Domingos, R. F.; Pinheiro, J. P.; Masini, J. C.; Algal Res. 2017, 24, 347.

112. Li, M.; Li, Da-W.; Guangli, X.; Long, Yi-T.; Curr. Opin. Electrochem. 2017, 3, 143 .

113. Cadkovaa, M.; Kovarovaa, A.; Dvorakovaa, V.; Metelkab, R.; Bilkovaa, Z.; Koreckaa, L.; Talanta 2018, 182, 115.

114. Parat, C.; Authier, L.; Aguilar, D.; Companys, E.; Puy, J.; Galceran, J.; Potin-Gautier, M.; Analista 2011, 136, 4337.

115. Parat, C.; Aguilar, D.; Authier, L.; Potin-Gautier, M.; Companys, E.; Puy, J.; Galceran, J.; Electroanalysis 2011, 23, 627.

116. Domingos, R. F.; Carreira, S.; Galceran, J.; Salaün, P.; Pinheiro, J. P.; Anal. Chim. Acta. 2016, 920, 29. 